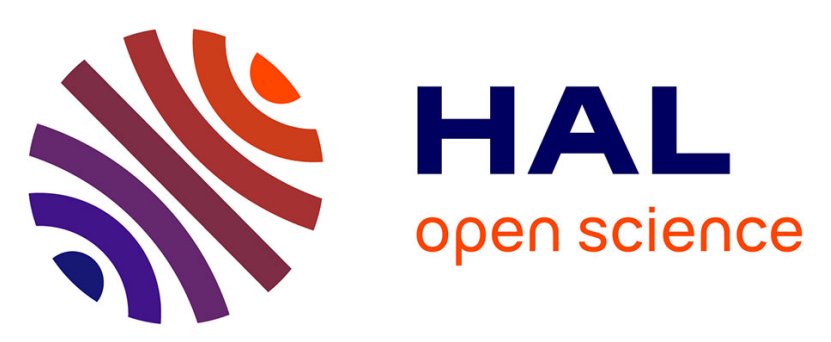

\title{
Efficient high order semi-implicit time discretization and local discontinuous Galerkin methods for highly nonlinear PDEs
}

\author{
Ruihan Guo, Francis Filbet, Yan Xu
}

\section{- To cite this version:}

Ruihan Guo, Francis Filbet, Yan Xu. Efficient high order semi-implicit time discretization and local discontinuous Galerkin methods for highly nonlinear PDEs. Journal of Scientific Computing, 2016, 68 (3), pp.1029-1054. hal-01203753v2

HAL Id: hal-01203753

https://hal.science/hal-01203753v2

Submitted on 8 Jan 2016

HAL is a multi-disciplinary open access archive for the deposit and dissemination of scientific research documents, whether they are published or not. The documents may come from teaching and research institutions in France or abroad, or from public or private research centers.
L'archive ouverte pluridisciplinaire HAL, est destinée au dépôt et à la diffusion de documents scientifiques de niveau recherche, publiés ou non, émanant des établissements d'enseignement et de recherche français ou étrangers, des laboratoires publics ou privés. 


\title{
Efficient high order semi-implicit time discretization and local discontinuous Galerkin methods for highly nonlinear PDEs
}

\author{
Ruihan Guo, Francis Filbet ${ }^{\dagger}$, Yan $\mathrm{Xu}^{\ddagger}$
}

\begin{abstract}
In this paper, we develop a high order semi-implicit time discretization method for highly nonlinear PDEs, which consist of the surface diffusion and Willmore flow of graphs, the Cahn-Hilliard equation and the Allen-Cahn/Cahn-Hilliard system. These PDEs are high order in spatial derivatives, which motivates us to develop implicit or semi-implicit time marching methods to relax the severe time step restriction for stability of explicit methods. In addition, these PDEs are also highly nonlinear, fully implicit methods will incredibly increase the difficulty of implementation. In particular, we can not well separate the stiff and non-stiff components for these problems, which leads to traditional implicit-explicit methods nearly meaningless. In this paper, a high order semi-implicit time marching method and the local discontinuous Galerkin (LDG) spatial method are coupled together to achieve high order accuracy in both space and time, and to enhance the efficiency of the proposed approaches, the resulting linear or nonlinear algebraic systems are solved by multigrid solver. Specially, we develop a first order fully discrete LDG scheme for the Allen-Cahn/Cahn-Hilliard system and prove the unconditional energy stability. Numerical simulation results in one and two dimensions are presented to illustrate that the combination of the LDG method for
\end{abstract}

*Institut Camille Jordan, Université Claude Bernard Lyon I, 43 boulevard 11 novembre 1918, 69622 Villeurbanne cedex, France. Email: rguo@math.univ-lyon1.fr.

†Institut de Mathématiques de Toulouse, Université Paul Sabatier Toulouse III, 118 route de Narbonne, 31062 Toulouse cedex, France. Email: francis.filbet@math.univ-toulouse.fr.

${ }^{\ddagger}$ School of Mathematical Sciences, University of Science and Technology of China, Hefei, Anhui 230026, P.R. China. Email: yxu@ustc.edu.cn. Research supported by NSFC grant No. 11371342, 11426236. 
spatial approximation, semi-implicit temporal integration with the multigrid solver provides a practical and efficient approach when solving this family of problems.

Key words: semi-implicit time marching method, local discontinuous Galerkin method, multigrid, surface diffusion and Willmore flow of graphs, Cahn-Hilliard equation, Allen-Cahn/Cahn-Hilliard system

\section{Introduction}

In this paper, we consider efficient high order semi-implicit time discretization and the local discontinuous Galerkin (LDG) method for time dependent highly nonlinear partial differential equations (PDEs) containing high order spatial derivatives.

The discontinuous Galerkin (DG) method is a class of finite element methods, in which using a completely discontinuous piecewise polynomials as the numerical solution and the test spaces. Reed and Hill [15] first designed it as a method for solving first order linear transport equation. Cockburn et al. later extended the DG method to solve nonlinear hyperbolic conservation laws in a series of papers $[3,4,5,6]$.

It is difficult to apply the DG method directly to PDEs containing higher order spatial derivatives, therefore the LDG method was introduced. The idea of the LDG method is to rewrite the equations with higher order derivatives as a first order system, then apply the DG method to the system. The first LDG method was constructed by Cockburn and Shu in [7] for solving a convection diffusion equation (containing second derivatives). Their work was motivated by the successful numerical experiments of Bassi and Rebay [1] for the compressible Navier-Stokes equations. For a detailed description about the LDG methods for high order time-dependent PDEs, we refer the readers to the review paper [23]. A common feature of these LDG methods is that stability can be proved for quite general nonlinear cases. DG and LDG methods also have several attractive properties, such as easy parallelization, easy adaptivity and simple treatment of boundary conditions. The most important property of DG and LDG methods is high order accurate, which motivates us to develop high order temporal accuracy scheme to get the goal of obtaining high order accuracy in both space and time together with robust stability conditions.

By the method of lines, the application of the LDG method for spatial variables for a partial differential equation will generate a large coupled system of ordinary differential equations (ODEs). The development of a suitable ODEs solver attracted a lot of attention 
in the last decades. Explicit high order nonlinearly stable Runge-Kutta methods are efficient for hyperbolic problems or convection dominated problems. However, if the PDEs contain high order spatial derivatives with coefficients not very small, then explicit temporal methods suffer from severe time step restrictions for stability, of the form $\Delta t \leq C \Delta x^{p}$, where $p$ is the order of the PDE. It would therefore be desirable to develop implicit or semi-implicit time discretization techniques to alleviate this problem, especially for long time simulations.

In [19], three different time discretization techniques for solving the stiff ODEs resulting from an LDG spatial discretization to PDEs with higher order spatial derivatives were explored. These are the semi-implicit spectral deferred correction (SDC) method, the additive Runge-Kutta (ARK) method and the exponential time differencing (ETD) method, which are all validated to be efficient. However, these three methods are mainly efficient for a problem with easily separate stiff and non-stiff components, which treating the non-stiff terms explicitly and the stiff terms implicitly. Actually, it is not always easy to separate stiff and non-stiff components, for example, for the surface diffusion and Willmore flow of graphs, the Cahn-Hilliard equation with degenerate mobility and the Allen-Cahn/Cahn-Hilliard system with degenerate mobility, which are all highly nonlinear and containing higher order spatial derivatives. In such cases, one usually relies on fully implicit schemes. However, fully implicit schemes have the disadvantage of difficult implementation and poor stability properties, especially for fully nonlinear problems.

The surface diffusion and Willmore flow of graphs are both highly nonlinear fourth-order PDEs. Smereka [16] developed a splitting technique for the surface diffusion of graphs, which was effective to stabilize numerical schemes but it may affect the numerical accuracy. In [8], a first order semi-implicit numerical scheme for the Willmore flow of graphs based on a finite element method was presented. Various unconditionally stable first order $[10,11]$ temporal discretization schemes have been developed for the Cahn-Hilliard equation, based on the convex splitting technique. These schemes are only first order accurate, and for the CahnHilliard equation with constant mobility, it is easy to extend to higher order accurate ones by the methods introduced in [21], but for the Cahn-Hilliard equation with degenerate mobility, high order temporal schemes are very difficult to derive. There have been limited numerical simulations works in the existing literature for efficient semi-implicit time marching method for solving the Allen-Cahn/Cahn-Hilliard system, and it would therefore be desirable to develop high order semi-implicit schemes for the system.

In this paper, we focus on high order semi-implicit time marching methods for PDEs with 
high order spatial derivatives and highly nonlinear, i.e. the stiff and non-stiff components can not be well separated. Coupled with the LDG spatial discretization, we will construct a semi-implicit fully discrete scheme for the surface diffusion and Willmore flow of graphs, the Cahn-Hilliard equation with degenerate mobility and the Allen-Cahn/Cahn-Hilliard system, which is high order accurate in both space and time. Obviously, it requires to solve system of linear or nonlinear equations at each time step. Traditional iterative solution methods such as Gauss-Seidel method suffers from slow convergence rates, especially for larger system. To enhance the efficiency of the proposed approach, the multigrid solver is employed to solve the algebraic equations at each time step.

The outline of this paper is as follows. In Section 2, we give a description of the high order semi-implicit time marching method. Section 3 is devoted to the application of LDG method and the semi-implicit time marching method for a series of highly nonlinear PDEs with higher order spatial derivatives. Numerical examples are also presented, testing the performance of the time marching method coupled with the LDG spatial discretization for these PDEs, including the surface diffusion and Willmore flow of graphs, the Cahn-Hilliard equation with degenerate mobility and the Allen-Cahn/Cahn-Hilliard system. Finally we give concluding remarks in Section 4.

\section{The high order semi-implicit time marching method}

The surface diffusion and Willmore flow of graphs, the Cahn-Hilliard equation and the Allen-Cahn/Cahn-Hilliard system are all PDEs of highly nonlinear, and the stiff and nonstiff components for these problems can not be well separated. After the LDG spatial discretization for these problems, we can get an ODEs of the form

$$
\left\{\begin{array}{l}
\frac{d u}{d t}(t)=\mathcal{H}(t, u(t), u(t)), \\
u\left(t_{0}\right)=u_{0}
\end{array}\right.
$$

where $m \in \mathbb{N}, u(t) \in \mathbb{R}^{m}, \mathcal{H}: \mathbb{R} \times \mathbb{R}^{m} \times \mathbb{R}^{m} \rightarrow \mathbb{R}^{m}$ and $\mathcal{H} \in C^{1}\left(\mathbb{R} \times \mathbb{R}^{m} \times \mathbb{R}^{m}\right)$, and the dependence on the second argument of $\mathcal{H}$ is non-stiff, while the dependence on the third argument is stiff. In this section, we will devote to developing a high order semi-implicit time marching method to solve (2.1). 


\subsection{The partitioned Runge-Kutta methods}

Boscarino et al. presented a new class of semi-implicit Runge-Kutta methods in [2], which was based on the partitioned Runge-Kutta methods. Therefore, in order to introduce the semi-implicit Runge-Kutta method, we will first give a simple description for the partitioned Runge-Kutta methods. We consider autonomous differential equations in the partitioned form,

$$
\left\{\begin{array}{l}
\frac{d y}{d t}(t)=\mathcal{F}(y(t), z(t)), \\
\frac{d z}{d t}(t)=\mathcal{G}(y(t), z(t)),
\end{array}\right.
$$

where $y(t) \in \mathbb{R}^{m}, z(t) \in \mathbb{R}^{n}, \mathcal{F}: \mathbb{R}^{m} \times \mathbb{R}^{n} \rightarrow \mathbb{R}^{m}, \mathcal{G}: \mathbb{R}^{m} \times \mathbb{R}^{n} \rightarrow \mathbb{R}^{n}$ and $\mathcal{F}, \mathcal{G} \in \mathcal{C}^{1}\left(\mathbb{R}^{m} \times \mathbb{R}^{n}\right)$, which are sufficient to guarantee local existence and uniqueness of the solution to (2.2). $y\left(t_{0}\right)=y_{0}, z\left(t_{0}\right)=z_{0}$ are the initial conditions.

Then we can express the partitioned Runge-Kutta methods by applying two different Runge-Kutta methods as the following Butcher tableau:

$$
\begin{array}{l|l}
\hat{c} & \hat{A} \\
\hline & \hat{b}^{T}
\end{array} \quad \begin{array}{c|c}
A \\
\hline
\end{array}
$$

In the above tableau, the pair $(\hat{A} \mid \hat{b})$ determines explicit Runge-Kutta methods and $(A \mid b)$ defines implicit Runge-Kutta methods, which means that the first variable $y(t)$ is treated by explicit method and the second one $z(t)$ is treated by implicit method. The Butcher coefficients $\hat{A}=\left(\hat{a}_{i, j}\right), A=\left(a_{i, j}\right) \in \mathbb{R}^{s \times s}, \hat{b}^{T}=\left(\hat{b}_{1}, \ldots, \hat{b}_{s}\right), b^{T}=\left(b_{1}, \ldots, b_{s}\right), \hat{c}=\left(\hat{c}_{1}, \ldots, \hat{c}_{s}\right)$ and $c=\left(c_{1}, \ldots, c_{s}\right)$ are constrained by order of accuracy and stability considerations.

For practical reasons, in order to simplify the computations, we consider that $\hat{A}$ is a strictly lower triangular matrix and $A$ is a lower triangular matrix for the implicit part. In addition, the coefficients satisfy:

$$
\hat{c}_{i}=\sum_{j=1}^{i-1} \hat{a}_{i, j}, \quad \text { and } \quad c_{i}=\sum_{j=1}^{i} a_{i, j}, \quad \text { for } \quad 1 \leqslant i \leqslant s .
$$

By applying the partitioned Runge-Kutta time marching method, the solution of the autonomous system (2.2) advanced from time $t^{n}$ to $t^{n+1}=t^{n}+\Delta t$ is given by

$$
\left\{\begin{array}{l}
k_{i}=\mathcal{F}\left(y^{n}+\Delta t \sum_{j=1}^{i-1} \hat{a}_{i, j} k_{j}, z^{n}+\Delta t \sum_{j=1}^{i} a_{i, j} l_{j}\right), \quad 1 \leqslant i \leqslant s, \\
l_{i}=\mathcal{G}\left(y^{n}+\Delta t \sum_{j=1}^{i-1} \hat{a}_{i, j} k_{j}, z^{n}+\Delta t \sum_{j=1}^{i} a_{i, j} l_{j}\right), \quad 1 \leqslant i \leqslant s,
\end{array}\right.
$$


and we can calculate $y^{n+1}$ and $z^{n+1}$ as follows

$$
\left\{\begin{array}{l}
y^{n+1}=y^{n}+\Delta t \sum_{i=1}^{s} \hat{b}_{i} k_{i} \\
z^{n+1}=z^{n}+\Delta t \sum_{i=1}^{s} b_{i} l_{i} .
\end{array}\right.
$$

\subsection{The semi-implicit Runge-Kutta methods}

Now let us assume that for every $i \in\{1, \ldots, s\}, b_{i}=\hat{b}_{i}$ in $(2.4)$.

After an overview of the partitioned Runge-Kutta methods, we will pay special attention to the high order semi-implicit Runge-Kutta methods in this subsection. Our goal is to develop a high order semi-implicit time marching method for equation (2.1), but not fully implicit scheme. To derive a semi-implicit Runge-Kutta scheme, we first rewrite the non autonomous differential equation (2.1) as an autonomous system where we double the number of variable, that is,

$$
\begin{cases}\frac{d}{d t}\left(\begin{array}{l}
t \\
u(t)
\end{array}\right) & =\left(\begin{array}{l}
1 \\
\mathcal{H}(t, u(t), u(t))
\end{array}\right), \\
\frac{d u(t)}{d t} & =\mathcal{H}(t, u(t), u(t)) .\end{cases}
$$

This system now corresponds to an autonomous partitioned system $(2.3)$, with $y(t)=$ $(t, u(t)), \mathcal{F}=(1, \mathcal{H})$ and $z(t)=u(t), \mathcal{G}=\mathcal{H}$ and $y\left(t_{0}\right)=\left(t_{0}, u_{0}\right), z\left(t_{0}\right)=u_{0}$ are the initial conditions. Applying the partitioned Runge-Kutta scheme (2.3)-(2.4) to system (2.5), we can get a high order semi-implicit Runge-Kutta method for (2.1): the first component of the first equation (2.5) only gives

$$
\hat{c}_{i}=\sum_{j=1}^{i} \hat{a}_{i, j}
$$

whereas the second component of the first equation and the second equation of (2.5) are identical, which gives the following semi-implicit scheme

$$
k_{i}=\mathcal{H}\left(t^{n}+\hat{c}_{i} \Delta t, u^{n}+\Delta t \sum_{j=1}^{i-1} \hat{a}_{i, j} k_{j}, u^{n}+\Delta t \sum_{j=1}^{i} a_{i, j} k_{j}\right), \quad 1 \leqslant i \leqslant s .
$$

It is worth to mention here that $k_{i}$ is defined implicitely since $a_{i, i} \neq 0$. Therefore, starting from $u^{n}$, we give the algorithm to calculate $u^{n+1}$ in the following.

1. Set for $i=1, \ldots, s$,

$$
U_{i}=u^{n}+\Delta t \sum_{j=1}^{i-1} \hat{a}_{i, j} k_{j}
$$




$$
V_{i}=u^{n}+\Delta t \sum_{j=1}^{i} a_{i, j} k_{j}
$$

2. For $i=1, \ldots, s$, compute

$$
k_{i}=\mathcal{H}\left(t^{n}+\hat{c}_{i} \Delta t, U_{i}, V_{i}\right) .
$$

3. Update the numerical solution $u^{n+1}$ as

$$
u^{n+1}=u^{n}+\Delta t \sum_{i=1}^{s} b_{i} k_{i}
$$

Remark 2.1. Observe that the augmented system (2.5) is only used to construct the partitioned Runge-Kutta method as for (2.2). Since the second component of the first equation of (2.5) and the second equation of (2.5) are the same, we only evaluate $\mathcal{H}$ once at each stage and $l_{i}$ corresponds to the second component of $k_{i}$, for any $i \in\{1, \ldots, n\}$. Furthermore, since $b_{i}=\hat{b}_{i}$, we also have in (2.4) that $z^{n+1}$ corresponds to the second component of $y^{n+1}$.

By algorithm (2.6)-(2.8), the second variable of equation (2.1) is treated explicitly and the third one is treated implicitly. Obviously, it is necessary to solve system of linear or nonlinear algebraic equations (2.7) at each time step. The overall performance highly depends on the performance of the solver. Traditional iterative solution methods such as Gauss-Seidel method suffers from slow convergence rates, especially for large systems. To enhance the efficiency of the high order semi-implicit time marching method, we will apply the multigrid solver to solve algebraic equations (2.7) in this paper. And for a detailed description of the multigrid solver, we refer the readers to Trottenberg et al. [17].

In this paper, we focus on a second order L-stable scheme and a third order one, which

\begin{tabular}{|c|c|c|}
\hline 0 & $\gamma$ & $\gamma$ \\
\hline 1 & $1-\gamma$ & $1-2 \gamma$ \\
\hline $1 / 2 \quad 1 / 2$ & & $1 / 2$ \\
\hline
\end{tabular}
were introduced in the context of hyperbolic systems with stiff relaxation in [14]. The second order L-stable is given in the following form

with $\gamma=1-1 / \sqrt{2}$. While for the third order L-stable scheme, the corresponding Butcher 
tableau is given by

\begin{tabular}{|c|c|c|c|c|c|c|c|c|c|}
\hline 0 & 0 & 0 & 0 & 0 & $\alpha$ & $\alpha$ & 0 & 0 & 0 \\
\hline 0 & 0 & 0 & 0 & 0 & 0 & $-\alpha$ & $\alpha$ & 0 & 0 \\
\hline 1 & 0 & 1 & 0 & 0 & 1 & 0 & $1-\alpha$ & $\alpha$ & 0 \\
\hline \multirow[t]{2}{*}{$1 / 2$} & 0 & $1 / 4$ & $1 / 4$ & 0 & $1 / 2$ & $\beta$ & $\eta$ & $1 / 2-\beta-\eta-\alpha$ & $\alpha$ \\
\hline & 0 & $1 / 6$ & $1 / 6$ & $2 / 3$ & & 0 & $1 / 6$ & $1 / 6$ & $2 / 3$ \\
\hline
\end{tabular}

with $\alpha=0.24169426078821, \beta=\alpha / 4$ and $\eta=0.12915286960590$.

\section{Applications of LDG method and semi-implicit time marching methods}

In this section, we perform numerical experiments of the LDG scheme coupled with the proposed high order semi-implicit time marching method for the surface diffusion and Willmore flow of graphs, the Cahn-Hilliard equation and the Allen-Cahn/Cahn-Hilliard system. To enhance the efficiency of the proposed approach, the multigrid solver is used to solve the algebraic equations at each time step. We present some accuracy tests to show that the proposed spatial and time discretization methods can achieve high order accuracy in both space and time. All the computations are performed in double precision and on uniform spatial meshes.

\subsection{The surface diffusion and Willmore flow of graphs}

In this subsection, we consider LDG spatial discretization coupled with high order semiimplicit Runge-Kutta method (2.6)-(2.8) for the surface diffusion of graphs

$$
\frac{\partial u}{\partial t}+\nabla \cdot\left(Q\left(\boldsymbol{I}-\frac{\nabla u \otimes \nabla u}{Q^{2}}\right) \nabla H\right)=0
$$

and Willmore flow of graphs

$$
\frac{\partial u}{\partial t}+Q \nabla \cdot\left(\frac{1}{Q}\left(\boldsymbol{I}-\frac{\nabla u \otimes \nabla u}{Q^{2}}\right) \nabla(Q H)\right)-\frac{1}{2} Q \nabla \cdot\left(\frac{H^{2}}{Q} \nabla u\right)=0,
$$

where $Q$ is the area element

$$
Q=\sqrt{1+|\nabla u|^{2}}
$$


and $H$ is mean curvature of the domain boundary $\Gamma$

$$
H=\nabla \cdot\left(\frac{\nabla u}{Q}\right) \text {. }
$$

$\mathrm{Xu}$ and Shu [22] developed the LDG finite element methods for these two equations, which were high order accurate in space. However, the forward Euler method was applied for time discretization with a suitably small time step $\Delta t\left(\Delta t=O\left(\Delta x^{4}\right)\right)$ for stability, which was not efficient, especially for long time simulations. These two equations are both highly nonlinear, which increases the difficulty of developing semi-implicit time marching method, not to mention high order scheme. To achieve high order accuracy in time, we will apply the proposed semi-implicit Runge-Kutta method (2.6)-(2.8) to these two equations.

After the LDG spatial discretization, we can apply our semi-implicit Runge-Kutta scheme by writing the ODEs in the form (2.1) with $u$ the component treated explicitly, $v$ the component treated implicitly and

$$
\mathcal{H}_{S D}(t, u, v)=-\nabla \cdot\left(Q_{S D}(u)\left(\boldsymbol{I}-\frac{\nabla u \otimes \nabla u}{Q_{S D}(u)^{2}}\right) \nabla H_{S D}(u, v)\right)
$$

for surface diffusion of graphs (3.9), where

$$
Q_{S D}(u)=\sqrt{1+|\nabla u|^{2}}, \quad \text { and } \quad H_{S D}(u, v)=\nabla \cdot\left(\frac{\nabla v}{Q_{S D}(u)}\right) .
$$

While for Willmore flow of graphs $(3.10), \mathcal{H}$ is given as

$$
\begin{aligned}
\mathcal{H}_{W}(t, u, v) & =-Q_{W}(u) \nabla \cdot\left(\frac{1}{Q_{W}(u)}\left(\boldsymbol{I}-\frac{\nabla u \otimes \nabla u}{Q_{W}(u)^{2}}\right) \nabla\left(Q_{W}(u) H_{2, W}(u, v)\right)\right) \\
& +\frac{1}{2} Q_{W}(u) \nabla \cdot\left(\frac{H_{1, W}(u)^{2}}{Q_{W}(u)} \nabla v\right),
\end{aligned}
$$

where $Q_{W}(u)=\sqrt{1+|\nabla u|^{2}}$

$$
H_{2, W}(u, v)=\nabla \cdot\left(\frac{\nabla v}{Q_{W}(u)}\right) \text { and } H_{1, W}(u)=\nabla \cdot\left(\frac{\nabla u}{Q_{W}(u)}\right) .
$$

With the proposed space and time discretization methods, we will achieve schemes with high order accuracy in both space and time with a larger time step, i.e. $\Delta t=O(\Delta x)$. Next we will present some numerical experiments to validate the result.

\section{Example 3.1. Accuracy test for surface diffusion of graphs}

In this example, we consider the accuracy test for one-dimensional surface diffusion of graphs. We test our method taking the exact solution

$$
u(x, t)=0.05 \cos (t) \sin (x)
$$


for equation (3.9) with a source term $f$, which is a given function so that (3.11) is the exact solution. The computational domain is $[-\pi, \pi]$, with the periodic boundary condition. The time step is taken as $\Delta t=0.1 \Delta x$. When the piecewise $\mathcal{P}^{1}$ elements are used in the LDG method, the second order semi-implicit Runge-Kutta method is used for time integration, while for $\mathcal{P}^{2}$ approximation, we adopt the third order scheme. The $L^{2}$ and $L^{\infty}$ errors and the numerical orders of accuracy at time $T=0.5$ are contained in Table 3.1, which shows $(k+1)$-th order of accuracy in both $L^{2}$ and $L^{\infty}$ norms for $P^{k}$ approximation.

To demonstrate the optimal complexity (with respect to the grid size $\Delta x$ ) of the multigrid solver, we provide evidence that the multigrid convergence rate is independent of $\Delta x$ for $\mathcal{P}^{1}$ and $\mathcal{P}^{2}$ approximation, which is shown in Figure 3.1.

\begin{tabular}{|c|c|cc|cc|}
\hline & $N$ & $L^{2}$ error & order & $L^{\infty}$ error & order \\
\hline \multirow{4}{*}{$\mathcal{P}^{1}$} & 16 & $7.31 \mathrm{E}-04$ & - & $9.13 \mathrm{E}-04$ & - \\
& 32 & $1.82 \mathrm{E}-04$ & 2.00 & $2.30 \mathrm{E}-04$ & 1.99 \\
& 64 & $4.56 \mathrm{E}-05$ & 2.00 & $5.76 \mathrm{E}-05$ & 2.00 \\
& 128 & $1.14 \mathrm{E}-05$ & 2.00 & $1.44 \mathrm{E}-05$ & 2.00 \\
\hline \multirow{4}{*}{$\mathcal{P}^{2}$} & 16 & $2.29 \mathrm{E}-05$ & - & $2.69 \mathrm{E}-05$ & - \\
& 32 & $2.87 \mathrm{E}-06$ & 3.00 & $3.40 \mathrm{E}-06$ & 2.99 \\
& 64 & $3.59 \mathrm{E}-07$ & 3.00 & $4.26 \mathrm{E}-07$ & 3.00 \\
& 128 & $4.48 \mathrm{E}-08$ & 3.00 & $5.62 \mathrm{E}-08$ & 2.92 \\
\hline
\end{tabular}

Table 3.1: Accuracy test for surface diffusion of graphs (3.9) with the exact solution (3.11) at time $T=0.5$.

\section{Example 3.2. Positive perturbation for surface diffusion of graphs}

In this example, we consider the numerical solutions of the two-dimensional surface diffusion of graphs (3.9) with the initial condition

$$
u_{0}(x, y)=1+0.3 \min \left(1, \max \left(0,2-5 \sqrt{x^{2}+y^{2}}\right)\right)
$$

and periodic boundary conditions. The computational domain is $[-1,1] \times[-1,1]$. The $\mathcal{P}^{2}$ elements with $64 \times 64$ cells and third order semi-implicit Runge-Kutta method are taken for space and time discretization, respectively. The numerical solutions at time $T=0,0.0001$, 0.001 and 0.005 are presented in Figure 3.2, which shows statistically similar patterns in the numerical solution as those in $\mathrm{Xu}$ and $\mathrm{Shu}[22]$, but with the advantage of taking larger time step $(\Delta t=O(\Delta x))$ comparing with the explicit time marching method $\left(\Delta t=O\left(\Delta x^{4}\right)\right)$ in [22]. 


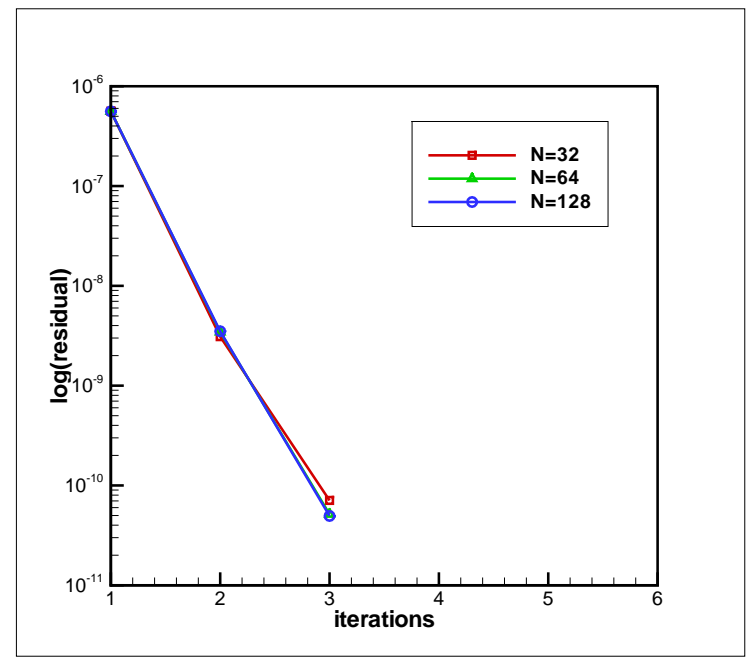

(a) $\mathcal{P}^{1}$ approximation

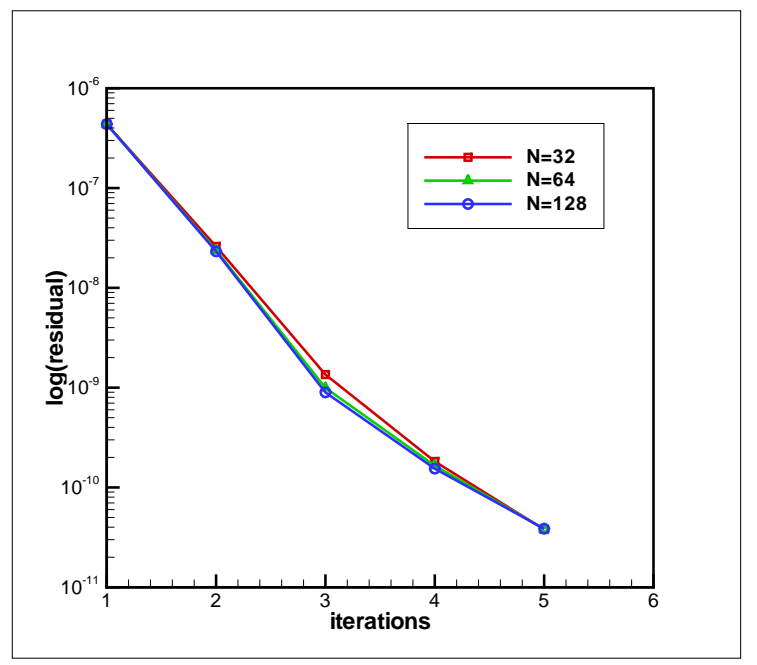

(b) $\mathcal{P}^{2}$ approximation

Figure 3.1: Convergence rates of multigrid solver with $\mathcal{P}^{1}$ and $\mathcal{P}^{2}$ approximation for surface diffusion of graphs.

We present in Figure 3.3 the time evolution of the $L^{2}$ norm of the numerical solution and its dissipation, i.e.

$$
\frac{d}{d t} \mathcal{E}_{S D}(t)=-\mathcal{I}_{S D}(t)
$$

where the functional $\mathcal{E}_{S D}(t)$ and the dissipation $\mathcal{I}_{S D}(t)$ are defined by

$$
\mathcal{E}_{S D}(t)=\frac{1}{2} \int_{\Omega} u^{2}(t, \boldsymbol{x}) d \boldsymbol{x}, \quad \mathcal{I}_{S D}(t)=\int_{\Omega} H^{2}(u(t, \boldsymbol{x})) d \boldsymbol{x} .
$$

The results show that our numerical scheme is stable with a lager time step $(\Delta t=O(\Delta x))$.

\section{Example 3.3. Accuracy test for Willmore flow of graphs}

In this example, we consider the accuracy test for one-dimensional Willmore flow of graphs. We test our scheme taking the exact solution

$$
u(x, t)=0.05 \cos (t) \sin (x)
$$

for equation (3.10) with a source term $f$, which is a given function so that (3.13) is the exact solution. The computational domain is $[-\pi, \pi]$, with the periodic boundary condition. The time step is taken as $\Delta t=0.1 \Delta x$. When the piecewise $\mathcal{P}^{1}$ elements are used in the LDG method, the second order semi-implicit Runge-Kutta method is used for time integration, while for $\mathcal{P}^{2}$ approximation, we adopt the third order scheme. The $L^{2}$ and $L^{\infty}$ errors and the numerical orders of accuracy at time $T=0.5$ are contained in Table 3.2, which shows 


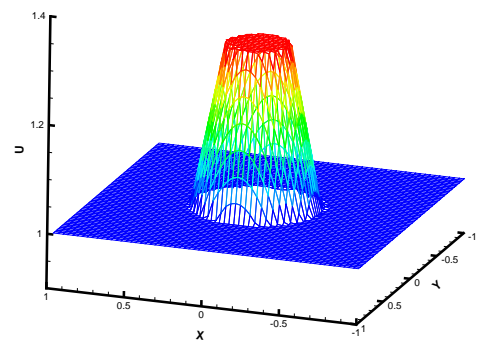

(a) $T=0$

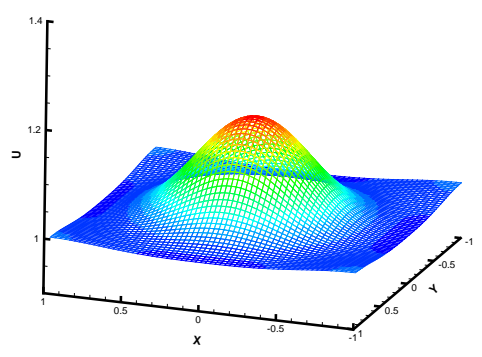

(c) $T=0.001$

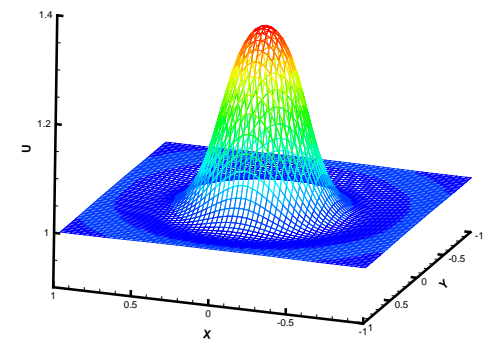

(b) $T=0.0001$

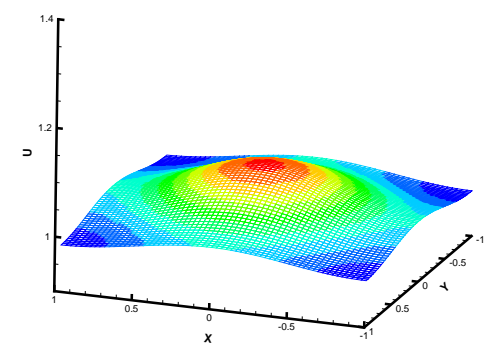

(d) $T=0.005$

Figure 3.2: Numerical solutions for the surface diffusion of graphs with the initial condition $(3.12)$.

$(k+1)$-th order of accuracy in both $L^{2}$ and $L^{\infty}$ norms for $\mathcal{P}^{k}$ approximation. Figure 3.4 shows the optimal complexity of the multigrid solver for the Willmore flow of graphs.

\section{Example 3.4. Sine perturbation for Willmore flow of graphs}

In this example, we consider the numerical solutions of the two-dimensional Willmore flow of graphs (3.10) in the square domain $\Omega=[-2,2] \times[-2,2]$ with the initial condition

$$
u_{0}(x, y)=0.25 \sin (\pi y)(0.25 \sin (\pi x)+0.5 \sin (3 \pi x))
$$

and periodic boundary condition. We use $\mathcal{P}^{2}$ elements with $64 \times 64$ cells and third order 


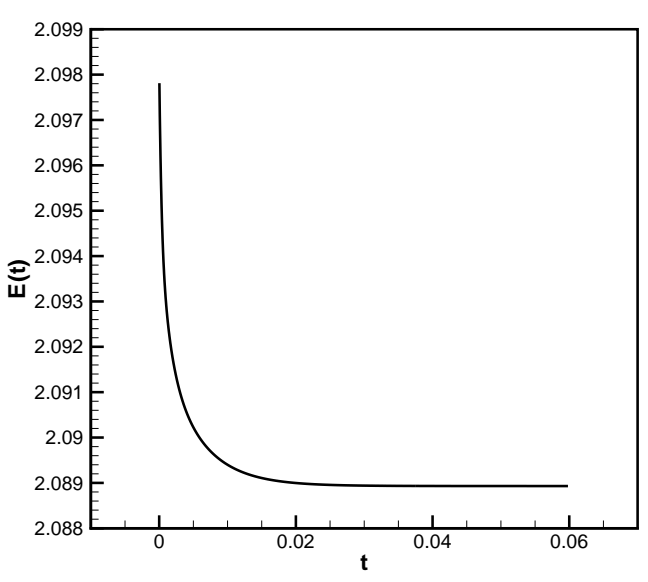

(a) functional $\mathcal{E}_{S D}(t)$

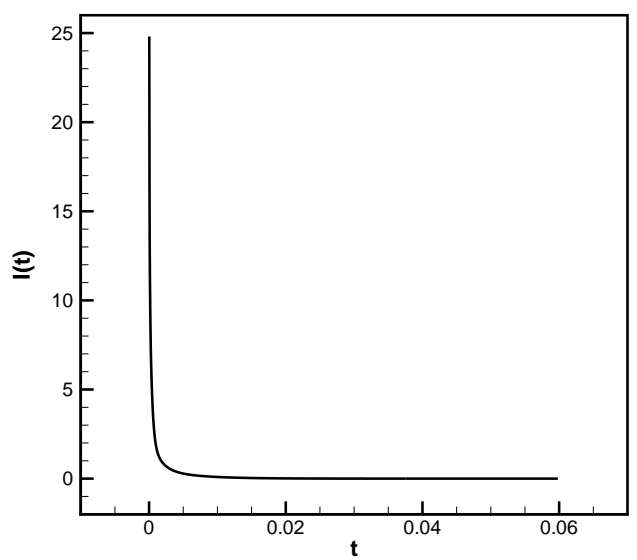

(b) dissipation $\mathcal{I}_{S D}(t)$

Figure 3.3: Evolution of the $L^{2}$ norm and the dissipation for surface diffusion of graphs

\begin{tabular}{|c|c|cc|cc|}
\hline & $N$ & $L^{2}$ error & order & $L^{\infty}$ error & order \\
\hline \multirow{4}{*}{$\mathcal{P}^{1}$} & 16 & $7.31 \mathrm{E}-04$ & - & $9.09 \mathrm{E}-04$ & - \\
& 32 & $1.82 \mathrm{E}-04$ & 2.00 & $2.28 \mathrm{E}-04$ & 1.99 \\
& 64 & $4.56 \mathrm{E}-05$ & 2.00 & $5.66 \mathrm{E}-05$ & 2.00 \\
& 128 & $1.14 \mathrm{E}-05$ & 2.00 & $1.39 \mathrm{E}-05$ & 2.02 \\
\hline \multirow{3}{*}{$\mathcal{P}^{2}$} & 16 & $2.29 \mathrm{E}-05$ & - & $2.69 \mathrm{E}-05$ & - \\
& 32 & $2.87 \mathrm{E}-06$ & 3.00 & $3.40 \mathrm{E}-06$ & 2.99 \\
& 64 & $3.59 \mathrm{E}-07$ & 3.00 & $4.26 \mathrm{E}-07$ & 2.99 \\
& 128 & $4.49 \mathrm{E}-08$ & 3.00 & $5.42 \mathrm{E}-08$ & 2.98 \\
\hline
\end{tabular}

Table 3.2: Accuracy test for Willmore flow of graphs (3.10) with the exact solution (3.13) at time $T=0.5$.

semi-implicit Runge-Kutta method to solve equation (3.10). The numerical solutions at time $T=0,0.0001,0.001$ and 0.01 are shown in Figure 3.5. With a larger time step of $\Delta t=O(\Delta x)$, our scheme gets the same results comparing the numerical calculations performed by Xu and Shu [22].

We present in Figure 3.6 the time evolution of the energy of the numerical solution and its dissipation, and the functional $\mathcal{E}_{W}(t)$ and the dissipation $\mathcal{I}_{W}(t)$ are defined by

$$
\mathcal{E}_{W}(t)=\frac{1}{2} \int_{\Omega} H^{2}(t, \boldsymbol{x}) Q(t, \boldsymbol{x}) d \boldsymbol{x}, \quad \mathcal{I}_{W}(t)=\int_{\Omega} \frac{(u(t, \boldsymbol{x}))^{2}}{Q(t, \boldsymbol{x})} d \boldsymbol{x} .
$$

From Figure 3.6, we can see that our numerical scheme is stable numerically, i.e. the discrete 


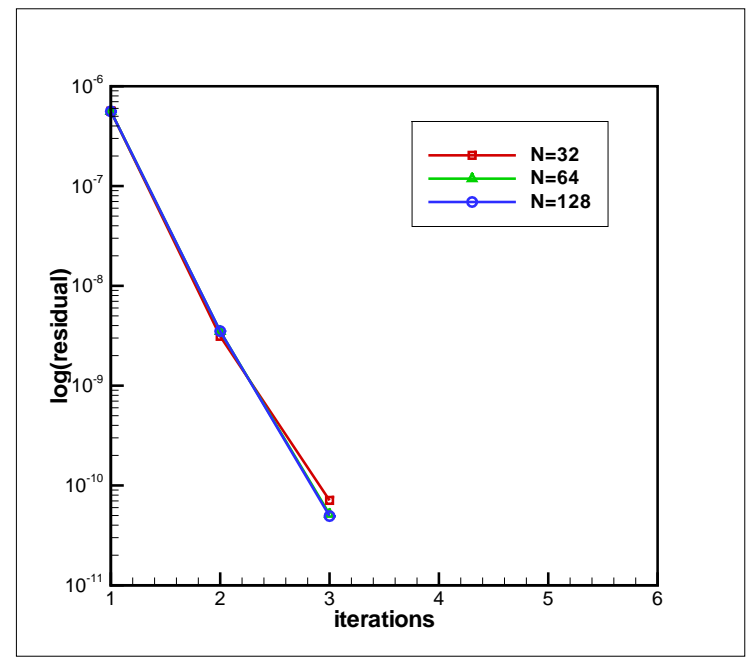

(a) $\mathcal{P}^{1}$ approximation

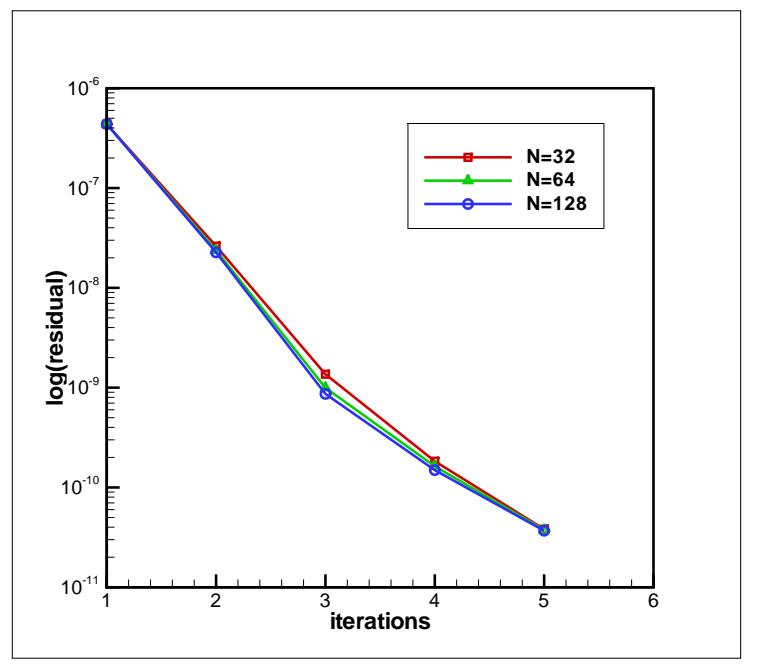

(b) $\mathcal{P}^{2}$ approximation

Figure 3.4: Convergence rates of multigrid solver with $\mathcal{P}^{1}$ and $\mathcal{P}^{2}$ approximation for Willmore flow of graphs.

energy is non-increasing about time.

\subsection{The Cahn-Hilliard equation}

In this subsection, we consider LDG spatial discretization coupled with high order semiimplicit Runge-Kutta method (2.6)-(2.8) for the Cahn-Hilliard equation

$$
\frac{\partial u}{\partial t}=\nabla \cdot\left[b(u) \nabla\left(-\gamma \Delta u+\Psi^{\prime}(u)\right)\right]
$$

where $\Psi(u)=\frac{1}{4}\left(u^{2}-1\right)^{2}, b(u)$ is the degenerate mobility, and $\gamma$ is a positive constant. Xia et al. [18] developed an LDG method for the Cahn-Hilliard equation and based on the consideration of high order in spatial derivative, Guo and Xu [11] constructed a semi-implicit convex splitting scheme for equation (3.15). The convex splitting scheme was unconditionally stable with a first order temporal accuracy, and the additive Runge-Kutta method was adopted in [11] to achieve high order accuracy in time for Cahn-Hilliard equation with constant mobility, i.e. $b(u)$ is constant. While for Cahn-Hilliard equation with degenerate mobility, Guo and $\mathrm{Xu}$ introduced the linearization scheme and fully implicit scheme to achieve high order temporal accuracy, but the implementation was difficult and the constraint of time step was still hard for these two methods. It would therefore be desirable to develop high order semi-implicit time marching method to solve the Cahn-Hilliard equation with degenerate mobility. 


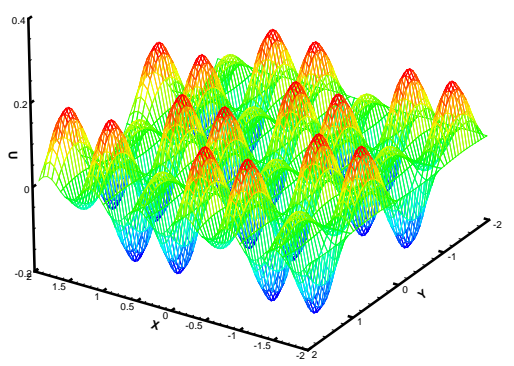

(a) $T=0$

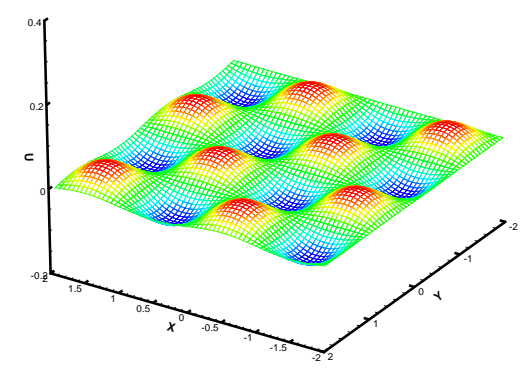

(c) $T=0.001$

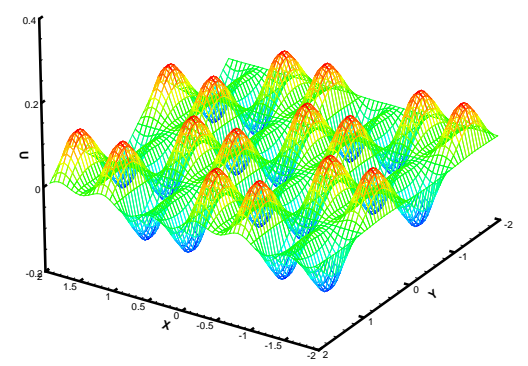

(b) $T=0.0001$

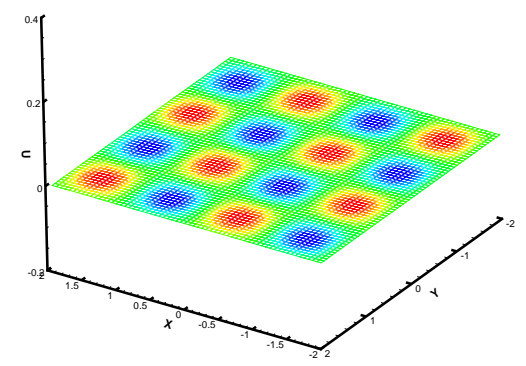

(d) $T=0.01$

Figure 3.5: Numerical solutions for the Willmore flow of graphs with the initial condition (3.14).

The convex splitting which was proposed in [11] is given in the following form:

$$
\frac{u^{n+1}-u^{n}}{\Delta t}=\nabla \cdot\left[b\left(u^{n}\right) \nabla\left(-\gamma \Delta u^{n+1}+\left(u^{n+1}\right)^{3}-u^{n}\right)\right] .
$$

Based on the unconditionally stable convex splitting scheme (3.16), and to apply our semiimplicit Runge-Kutta scheme, we rewrite the Cahn-Hilliard equation in the form of (2.1) with $u$ the component treated explicitly, $v$ the component treated implicitly, and

$$
\mathcal{H}_{C H}(t, u, v)=\nabla \cdot\left[b(u) \nabla\left(-\gamma \Delta v+v^{3}-u\right)\right] .
$$




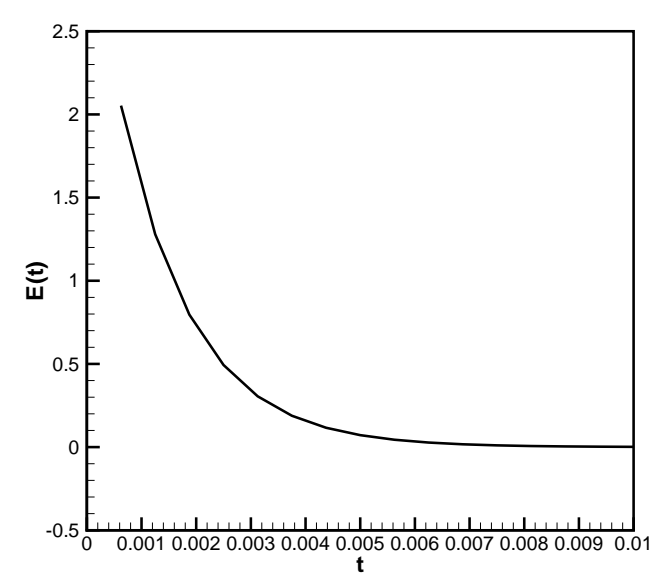

(a) functional $\mathcal{E}_{W}(t)$

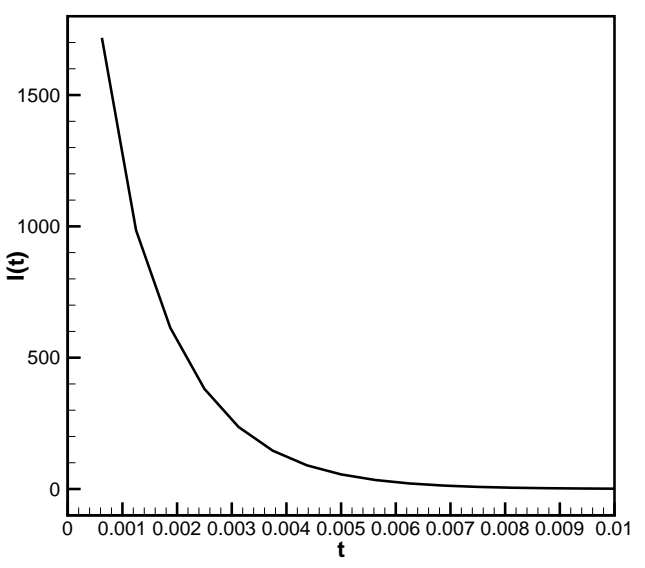

(b) dissipation $\mathcal{I}_{W}(t)$

Figure 3.6: Evolution of the energy and the dissipation for Willmore flow of graphs

Then we will get a stable semi-implicit scheme for the Cahn-Hilliard equation, which is high order accurate in both space and time.

\section{Example 3.5. Accuracy test for Cahn-Hilliard equation}

We consider the Cahn-Hilliard equation (3.15) with $b(u)=1-u^{2}, \gamma=1$ in the domain $\Omega=[-\pi, \pi]$ and with periodic boundary condition. We test our method taking the exact solution

$$
u(x, t)=e^{-t} \sin (x)
$$

for equation (3.15) with a source term $f$, which is a given function so that make the exact solution (3.18). The time step is taken as $\Delta t=0.1 \Delta x$. When the piecewise $\mathcal{P}^{1}$ elements are used in the LDG method, the second order semi-implicit Runge-Kutta method is used for time integration, while for $\mathcal{P}^{2}$ approximation, we adopt the third order scheme. Table 3.3 presents the $L^{2}$ and $L^{\infty}$ errors and the numerical orders of accuracy at time $T=0.5$, which shows $(k+1)$-th order of accuracy in both $L^{2}$ and $L^{\infty}$ norms for $\mathcal{P}^{k}$ approximation with larger time step comparing numerical methods in [18]. Figure 3.7 presents the optimal complexity of the multigrid solver for the Cahn-Hilliard equation, which shows that the convergence is independent of the grid size $\Delta x$.

\section{Example 3.6. Long time simulation for Cahn-Hilliard equation}

In the square domain $\Omega=[0,6.4] \times[0,6.4]$, we consider the Cahn-Hilliard equation (3.15) 


\begin{tabular}{|c|c|cc|cc|}
\hline & $N$ & $L^{2}$ error & order & $L^{\infty}$ error & order \\
\hline \multirow{4}{*}{$\mathcal{P}^{1}$} & 16 & $1.01 \mathrm{E}-02$ & - & $1.26 \mathrm{E}-02$ & - \\
& 32 & $2.52 \mathrm{E}-03$ & 2.00 & $3.16 \mathrm{E}-03$ & 2.00 \\
& 64 & $6.31 \mathrm{E}-04$ & 2.00 & $7.89 \mathrm{E}-04$ & 2.00 \\
& 128 & $1.57 \mathrm{E}-04$ & 2.00 & $1.96 \mathrm{E}-04$ & 2.00 \\
\hline \multirow{5}{*}{$\mathcal{P}^{2}$} & 16 & $3.18 \mathrm{E}-04$ & - & $3.75 \mathrm{E}-04$ & - \\
& 32 & $3.98 \mathrm{E}-05$ & 3.00 & $4.72 \mathrm{E}-05$ & 2.99 \\
& 64 & $5.00 \mathrm{E}-06$ & 2.99 & $5.98 \mathrm{E}-06$ & 2.98 \\
& 128 & $6.27 \mathrm{E}-07$ & 3.00 & $7.54 \mathrm{E}-07$ & 2.99 \\
\hline
\end{tabular}

Table 3.3: Accuracy test for the Cahn-Hilliard equation (3.15) with the exact solution (3.18) at time $T=0.5$.

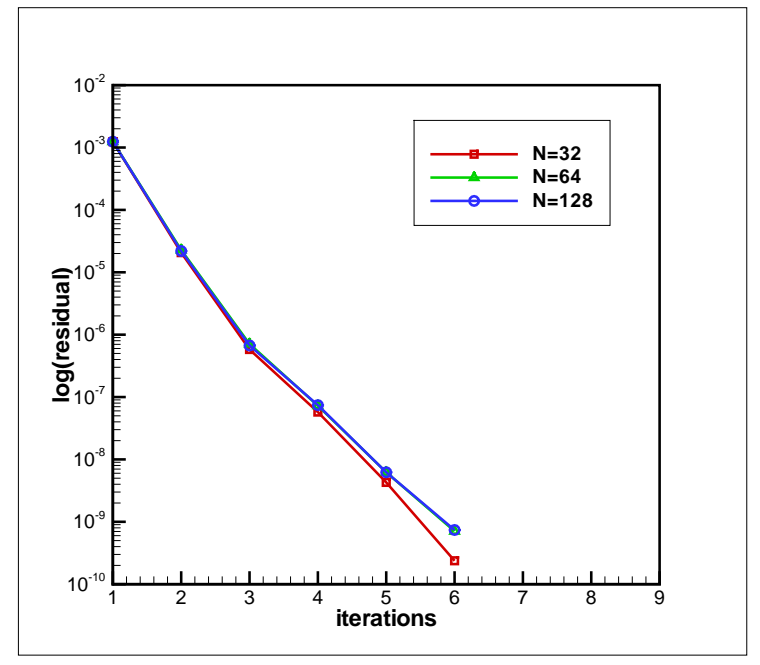

(a) $\mathcal{P}^{1}$ approximation

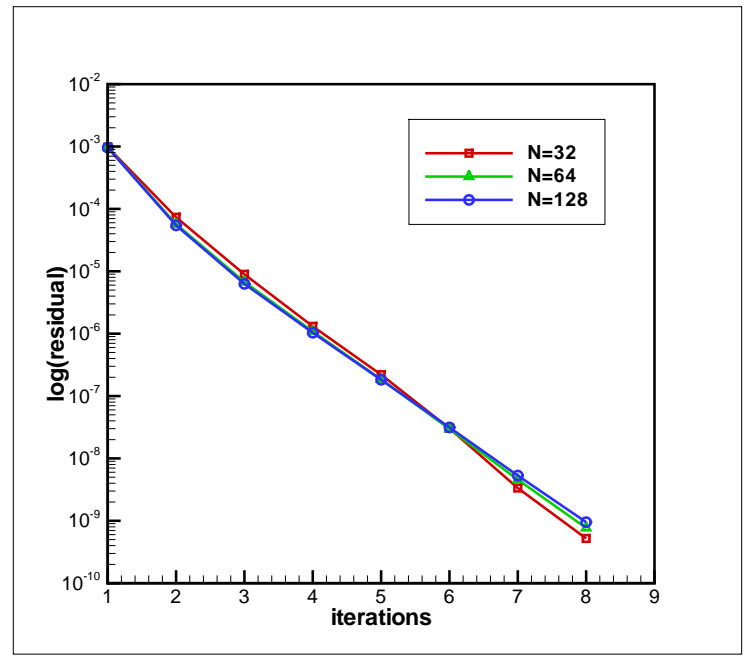

(b) $\mathcal{P}^{2}$ approximation

Figure 3.7: Convergence rates of multigrid solver with $\mathcal{P}^{1}$ and $\mathcal{P}^{2}$ approximation for CahnHilliard equation.

with

$$
\Psi^{\prime}(u)=u^{3}-u, \quad b(u)=\sqrt{(1+u)^{2}(1-u)^{2}+\gamma}, \quad \gamma=0.001
$$

and Neumann boundary condition, i.e.

$$
\frac{\partial u}{\partial \boldsymbol{\nu}}=b(u) \nabla\left[-\gamma \Delta u+\Psi^{\prime}(u)\right] \cdot \boldsymbol{\nu}=0, \quad \text { on } \partial \Omega .
$$

The initial data is a random field of values that are uniformly distributed about the average composition $\bar{u}=-0.05$, with amplitude 0.05 . 
We use the $\mathcal{P}^{2}$ element and third order semi-implicit Runge-Kutta method (3.17), which is based on the convex splitting scheme proposed in [11]. Figure 3.8 shows the contour lines of the numerical solution in some selected time levels. We present in Figure 3.9 the time evolution of the discrete energy of the numerical solution and its dissipation, i.e.

$$
\frac{d}{d t} \mathcal{E}_{C H}(t)=-\mathcal{I}_{C H}(t)
$$

where the functional $\mathcal{E}_{C H}$ and the dissipation $\mathcal{I}_{C H}$ are given by the following form

$$
\mathcal{E}_{C H}(t)=\int_{\Omega}\left[\frac{\gamma}{2}|\nabla u|^{2}+\Psi(u)\right] d \boldsymbol{x}, \quad \mathcal{I}_{C H}(t)=\int_{\Omega} b(u)|\nabla \mu|^{2} d \boldsymbol{x},
$$

with $\mu=-\gamma \Delta u+\Psi^{\prime}(u)$. The results show that our numerical scheme is stable.

This long time simulation example shows the capability of the LDG method, the high order semi-implicit temporal method and the multigrid solver for solving the Cahn-Hilliard equation with degenerate mobility.

\subsection{The Allen-Cahn/Cahn-Hilliard system}

In this subsection, we consider LDG spatial discretization coupled with high order semiimplicit time marching methods for the Allen-Cahn/Cahn-Hilliard system

$$
\left\{\begin{array}{l}
\frac{\partial u}{\partial t}=\nabla \cdot\left[b(u, v) \nabla\left(\Psi_{u}(u, v)-\gamma \Delta u\right)\right], \\
\rho \frac{\partial v}{\partial t}=-b(u, v)\left[\Psi_{v}(u, v)-\gamma \Delta v\right],
\end{array}\right.
$$

where

$$
\begin{aligned}
& \Psi_{u}(u, v)=\theta[\ln (u+v)-\ln (1-(u+v))+\ln (u-v)-\ln (1-(u-v))]+\frac{\alpha}{2}(1-2 u), \\
& \Psi_{v}(u, v)=\theta[\ln (u+v)-\ln (1-(u+v))-\ln (u-v)+\ln (1-(u-v))]-\beta v,
\end{aligned}
$$

the degenerate mobility is

$$
b(u, v)=u(1-u)\left(\frac{1}{4}-v^{2}\right)
$$

and the free energy is given by

$$
\mathcal{E}_{A C C H}(u, v)=\int_{\Omega}\left[\frac{\gamma}{2}\left(|\nabla u|^{2}+|\nabla v|^{2}\right)+\Psi(u, v)\right] d \boldsymbol{x} .
$$

The homogeneous free energy $\Psi(u, v)$ is

$$
\Psi_{A C C H}(u, v)=\theta[\Phi(u+v)+\Phi(u-v)]+\frac{1}{2}\left[\alpha u(1-u)-\beta v^{2}\right],
$$




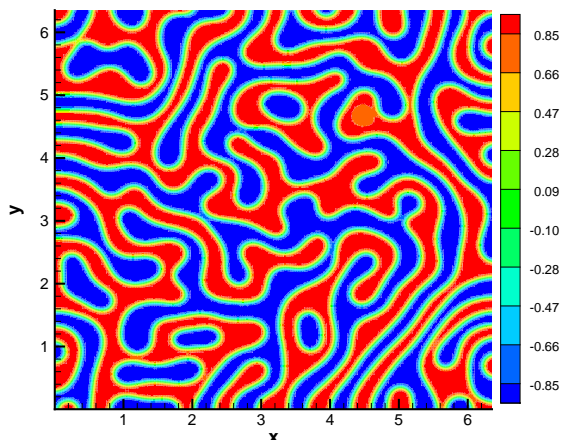

(a) $T=1$

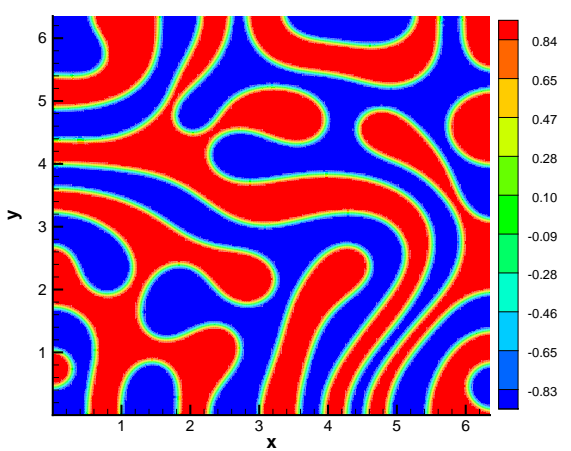

(c) $T=20$

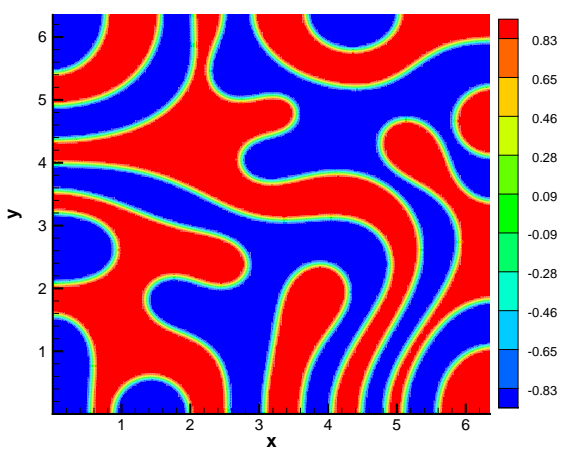

(e) $T=60$

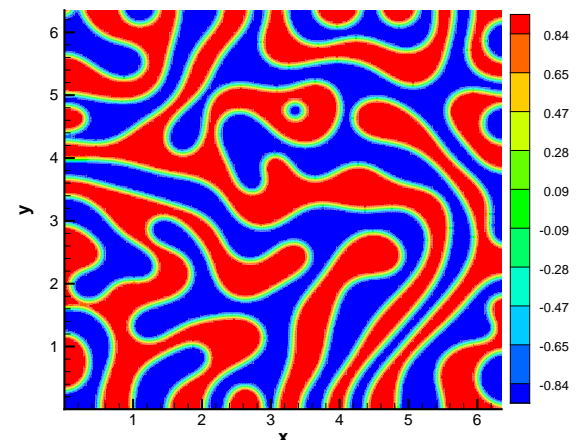

(b) $T=5$

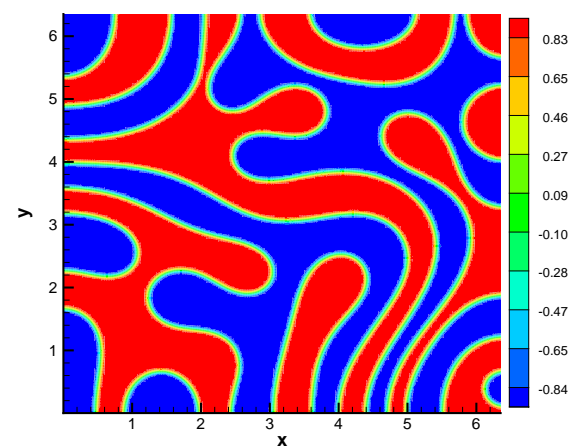

(d) $T=40$

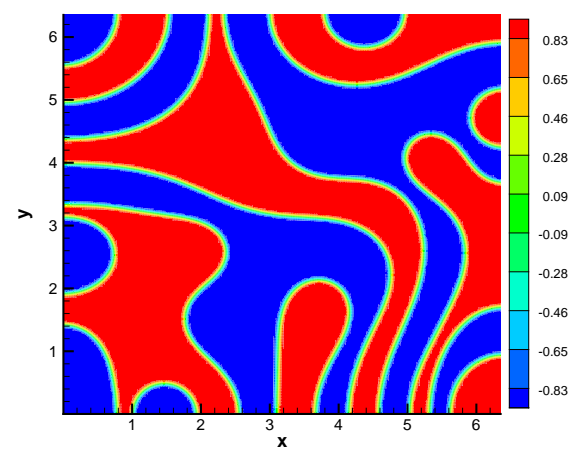

(f) $T=100$

Figure 3.8: The time evolution of the Cahn-Hilliard equation with $\mathcal{P}^{2}$ approximation on a $256 \times 256$ mesh. 


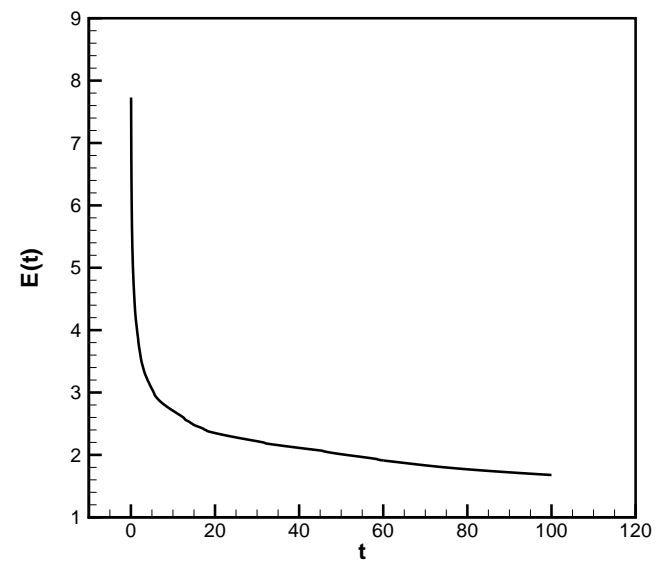

(a) functional $\mathcal{E}_{C H}$

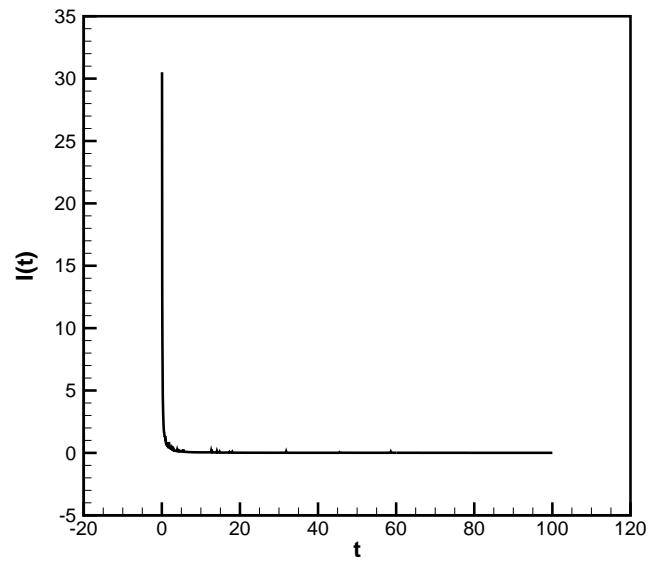

(b) dissipation $\mathcal{I}_{C H}$

Figure 3.9: Evolution of the energy and the dissipation for Cahn-Hilliard equation

where $\Phi(s)=s \ln s+(1-s) \ln (1-s)$.

Here $\theta$ represents the temperature, $\gamma$ is the coefficient of gradient energy and $\alpha, \beta$ are the coefficients of nearest and next-nearest pairwise energetic interactions. To close the system, we assume that the system (3.19) is supplemented with Neumann boundary conditions, i.e.

$$
\frac{\partial u}{\partial \boldsymbol{\nu}}=\frac{\partial v}{\partial \boldsymbol{\nu}}=b(u, v) \nabla\left(-\gamma \Delta u+\Psi_{u}(u, v)\right) \cdot \boldsymbol{\nu}=0, \quad \text { on } \partial \Omega .
$$

Xia et al. [20] developed an LDG method for the system (3.19), and in which an explicit time marching method was employed. However, the explicit method was not efficient because of its severe time step restriction $\left(\Delta t=O\left(\Delta x^{4}\right)\right)$ for stability, especially for long time simulations.

Here, we introduce the convex splitting method for time discretization coupled with LDG spatial discretization, to obtain a fully-discrete energy stable LDG scheme for a particular Allen-Cahn/Cahn-Hilliard system $(\theta=0)$. The corresponding semi-discrete convex splitting scheme is given as

$$
\left\{\begin{array}{l}
\frac{u^{n+1}-u^{n}}{\Delta t}=\nabla \cdot\left[b\left(u^{n}, v^{n}\right) \nabla\left(\Psi_{u}\left(u^{n}, v^{n}\right)-\gamma \Delta u^{n+1}\right)\right], \\
\rho \frac{v^{n+1}-v^{n}}{\Delta t}=-b\left(u^{n}, v^{n}\right)\left[\Psi_{v}\left(u^{n}, v^{n}\right)-\gamma \Delta v^{n+1}\right]
\end{array}\right.
$$

where

$$
\Psi_{u}\left(u^{n}, v^{n}\right)=\frac{\alpha}{2}\left(1-2 u^{n}\right), \quad \Psi_{v}\left(u^{n}, v^{n}\right)=-\beta v^{n}
$$


We consider a subdivision $\mathcal{T}_{h}$ of $\Omega$ with shape-regular elements $K$, and denote $\Gamma$ the union of the boundary faces of elements $K \in \mathcal{T}_{h}$, i.e. $\Gamma=\cup_{K \in \mathcal{T}_{h}} \partial K$, and $\Gamma_{0}=\Gamma \backslash \partial \Omega$. We set

$$
\begin{aligned}
V_{h} & =\left\{\varphi:\left.\quad \varphi\right|_{K} \in \mathcal{P}^{k}(K), \quad \forall K \in \mathcal{T}_{h}\right\}, \\
\Sigma_{h}^{d} & =\left\{\Phi=\left(\phi_{1}, \cdots, \phi_{d}\right)^{T}:\left.\quad \phi_{l}\right|_{K} \in \mathcal{P}^{k}(K), \quad l=1 \cdots d, \quad \forall K \in \mathcal{T}_{h}\right\},
\end{aligned}
$$

where $\mathcal{P}^{k}(K)$ is the space of polynomials of degree at most $k \geq 0$ on element $K$. Note that functions in $V_{h}$ and $\Sigma_{h}^{d}$ are allowed to be completely discontinuous across element interfaces.

To define the LDG method for scheme (3.23), we first rewrite it as a first order system

$$
\left\{\begin{array}{l}
\frac{u^{n+1}-u^{n}}{\Delta t}=\nabla \cdot s^{n+1} \\
\frac{v^{n+1}-v^{n}}{\Delta t}=-b\left(u^{n}, v^{n}\right)\left(-q_{2}^{n+1}+r_{2}^{n}\right) / \rho
\end{array}\right.
$$

with

$$
\left\{\begin{array}{l}
\boldsymbol{s}^{n+1}=b\left(u^{n}, v^{n}\right) \boldsymbol{p}^{n+1}, \\
\boldsymbol{p}^{n+1}=\nabla\left(-q_{1}^{n+1}+r_{1}^{n}\right), \\
q_{1}^{n+1}=\gamma \nabla \cdot \boldsymbol{w}_{1}^{n+1} \\
q_{2}^{n+1}=\gamma \nabla \cdot \boldsymbol{w}_{2}^{n+1} \\
\boldsymbol{w}_{1}^{n+1}=\nabla u^{n+1} \\
\boldsymbol{w}_{2}^{n+1}=\nabla v^{n+1} \\
r_{1}^{n}=\Psi_{u}\left(u^{n}, v^{n}\right), \\
r_{2}^{n}=\Psi_{v}\left(u^{n}, v^{n}\right) .
\end{array}\right.
$$

The LDG scheme for the semi-discrete scheme now consists in finding $u^{n+1}, v^{n+1}, q_{1}^{n+1}, q_{2}^{n+1}$, $r_{1}^{n}, r_{2}^{n} \in V_{h}$ and $\boldsymbol{s}^{n+1}, \boldsymbol{p}^{n+1}, \boldsymbol{w}_{1}^{n+1}, \boldsymbol{w}_{2}^{n+1} \in \Sigma_{h}^{d}$, such that, for all test functions $\varphi_{1}, \varphi_{2}, \varphi_{3}$, $\varphi_{4}, \varphi_{5}, \varphi_{6} \in V_{h}$ and $\boldsymbol{\theta}_{1}, \boldsymbol{\theta}_{2}, \boldsymbol{\theta}_{3}, \boldsymbol{\theta}_{4} \in \Sigma_{h}^{d}$, we have

$$
\left\{\begin{array}{l}
\int_{K} \frac{u^{n+1}-u^{n}}{\Delta t} \varphi_{1} d K=-\int_{K} s^{n+1} \cdot \nabla \varphi_{1} d K+\int_{\partial K} \widehat{\boldsymbol{s}}^{n+1} \cdot \boldsymbol{\nu} \varphi_{1} d s \\
\int_{K} \frac{v^{n+1}-v^{n}}{\Delta t} \varphi_{2} d K=-\int_{K} \frac{b\left(u^{n}, v^{n}\right)}{\rho}\left(r_{2}^{n}-q_{2}^{n+1}\right) \varphi_{2} d K
\end{array}\right.
$$

with

$$
\left\{\begin{array}{l}
\int_{K} \boldsymbol{s}^{n+1} \cdot \boldsymbol{\theta}_{1} d K=\int_{K} b\left(u^{n}, v^{n}\right) \boldsymbol{p}^{n+1} \cdot \boldsymbol{\theta}_{1} d K \\
\int_{K} \boldsymbol{p}^{n+1} \cdot \boldsymbol{\theta}_{2} d K=-\int_{K}\left(r_{1}^{n}-q_{1}^{n+1}\right) \nabla \cdot \boldsymbol{\theta}_{2} d K+\int_{\partial K}\left(\widehat{r}_{1}^{n}-\widehat{q}_{1}^{n+1}\right) \boldsymbol{\theta}_{2} \cdot \boldsymbol{\nu} d s,
\end{array}\right.
$$


and

$$
\left\{\begin{array}{l}
\int_{K} q_{1}^{n+1} \varphi_{3} d K=-\gamma \int_{K} \boldsymbol{w}_{1}^{n+1} \cdot \nabla \varphi_{3} d K+\gamma \int_{\partial K} \widehat{\boldsymbol{w}}_{1}^{n+1} \cdot \boldsymbol{\nu} \varphi_{3} d s \\
\int_{K} q_{2}^{n+1} \varphi_{4} d K=-\gamma \int_{K} \boldsymbol{w}_{2}^{n+1} \cdot \nabla \varphi_{4} d K+\gamma \int_{\partial K} \widehat{\boldsymbol{w}}_{2}^{n+1} \cdot \boldsymbol{\nu} \varphi_{4} d s
\end{array}\right.
$$

and

$$
\begin{aligned}
& \int_{K} \boldsymbol{w}_{1}^{n+1} \cdot \boldsymbol{\theta}_{3} d K=-\int_{K} u^{n+1} \nabla \cdot \boldsymbol{\theta}_{3} d K+\int_{\partial K} \widehat{u}^{n+1} \boldsymbol{\theta}_{3} \cdot \boldsymbol{\nu} d s \\
& \int_{K} \boldsymbol{w}_{2}^{n+1} \cdot \boldsymbol{\theta}_{4} d K=-\int_{K} v^{n+1} \nabla \cdot \boldsymbol{\theta}_{4} d K+\int_{\partial K} \widehat{v}^{n+1} \boldsymbol{\theta}_{4} \cdot \boldsymbol{\nu} d s, \\
& \int_{K} r_{1}^{n} \varphi_{5} d K=\int_{K} \Psi_{u}\left(u^{n}, v^{n}\right) \varphi_{5} d K \\
& \int_{K} r_{2}^{n} \varphi_{6} d K=\int_{K} \Psi_{v}\left(u^{n}, v^{n}\right) \varphi_{6} d K .
\end{aligned}
$$

Here $\widehat{\boldsymbol{s}}^{n+1}, \widehat{r}_{1}^{n}, \widehat{q}_{1}^{n+1}, \widehat{\boldsymbol{w}}_{1}^{n+1}, \widehat{\boldsymbol{w}}_{2}^{n+1}, \widehat{u}^{n+1}$ and $\widehat{v}^{n+1}$ are so-called "numerical fluxes". To complete the definition of LDG method, we need to define these numerical fluxes.

Let $e$ be an interior face shared by the "left" and "right" elements $K_{L}$ and $K_{R}$ and define the normal vectors $\boldsymbol{\nu}_{L}$ and $\boldsymbol{\nu}_{R}$ on $e$ pointing exterior to $K_{L}$ and $K_{R}$, respectively. For our purpose "left" and "right" can be uniquely defined for each face according to any fixed rule. For example, we choose $\boldsymbol{\nu}_{0}$ as a constant vector. The left element $K_{L}$ to the face $e$ requires that $\boldsymbol{\nu}_{L} \cdot \boldsymbol{\nu}_{0}<0$, and the right one $K_{R}$ requires $\boldsymbol{\nu}_{L} \cdot \boldsymbol{\nu}_{0} \geq 0$. If $\psi$ is a function on $K_{L}$ and $K_{R}$, but possibly discontinuous across $e$, let $\psi_{L}$ denote $\left.\left(\left.\psi\right|_{K_{L}}\right)\right|_{e}$ and $\psi_{R}$ denote $\left.\left(\left.\psi\right|_{K_{R}}\right)\right|_{e}$, the left and right trace, respectively. It turns out that we can take the simple alternating numerical fluxes to guarantee the energy stability, such as

$$
\left\{\begin{array}{l}
\widehat{\boldsymbol{s}}^{n+1}=\boldsymbol{s}_{L}^{n+1}, \\
\widehat{q}_{1}^{n+1}=q_{1, R}^{n+1}, \\
\widehat{r}_{1}^{n}=r_{1, R}^{n}, \\
\widehat{\boldsymbol{w}}_{1}^{n+1}=\boldsymbol{w}_{1, L}^{n+1}, \\
\widehat{u}^{n+1}=u_{R}^{n+1}, \\
\widehat{\boldsymbol{w}}_{2}^{n+1}=\boldsymbol{w}_{2, L}^{n+1}, \\
\widehat{v}^{n+1}=v_{R}^{n+1} .
\end{array}\right.
$$

We remark that the choice for the fluxes (3.28) is not unique. Considering the compactness of the stencil and the optimal accuracy, the crucial part is taking $\widehat{\boldsymbol{s}}^{n+1}$ and $\widehat{r}_{1}^{n}, \widehat{q}_{1}^{n+1}$ from opposite sides, $\widehat{\boldsymbol{w}}_{1}^{n+1}$ and $\widehat{u}^{n+1}$ from opposite sides, $\widehat{\boldsymbol{w}}_{2}^{n+1}$ and $\widehat{v}^{n+1}$ from opposite sides. 
It is easy to show that the LDG scheme is mass conservative. Next, we will prove the energy stability for fully-discrete LDG scheme (3.24) with the alternating numerical fluxes and Neumann boundary condition.

Proposition 3.1 (Energy stability for the fully-discrete LDG scheme). The solution to the LDG scheme (3.24) with the numerical fluxes (3.28) satisfies the energy stability

$$
\mathcal{E}_{h}\left(\boldsymbol{w}_{1}^{n+1}, \boldsymbol{w}_{2}^{n+1}, u^{n+1}, v^{n+1}\right)-\mathcal{E}_{h}\left(\boldsymbol{w}_{1}^{n}, \boldsymbol{w}_{2}^{n}, u^{n}, v^{n}\right) \leq 0
$$

where

$$
\mathcal{E}_{h}\left(\boldsymbol{w}_{1}, \boldsymbol{w}_{2}, u, v\right)=\int_{\Omega}\left(\frac{\gamma}{2}\left(\boldsymbol{w}_{1} \cdot \boldsymbol{w}_{1}+\boldsymbol{w}_{2} \cdot \boldsymbol{w}_{2}\right)+\Psi(u, v)\right) d \boldsymbol{x}
$$

Proof. For equations (3.27a) and (3.27b) of the LDG scheme, subtracting the equations at time level $t^{n}$ from the equations at time level $t^{n+1}$, respectively, we get

$$
\begin{aligned}
& \int_{K} \mathcal{D} \boldsymbol{w}_{1} \cdot \boldsymbol{\theta}_{3} d K=-\int_{K} \mathcal{D} u \nabla \cdot \boldsymbol{\theta}_{3} d K+\int_{\partial K} \mathcal{D} \widehat{u} \boldsymbol{\theta}_{3} \cdot \boldsymbol{\nu} d s \\
& \int_{K} \mathcal{D} \boldsymbol{w}_{2} \cdot \boldsymbol{\theta}_{4} d K=-\int_{K} \mathcal{D} v \nabla \cdot \boldsymbol{\theta}_{4} d K+\int_{\partial K} \mathcal{D} \widehat{v} \boldsymbol{\theta}_{4} \cdot \boldsymbol{\nu} d s
\end{aligned}
$$

where $\mathcal{D} \boldsymbol{w}_{1}=\boldsymbol{w}_{1}^{n+1}-\boldsymbol{w}_{1}^{n}, \mathcal{D} u=u^{n+1}-u^{n}, \mathcal{D} \boldsymbol{w}_{2}=\boldsymbol{w}_{2}^{n+1}-\boldsymbol{w}_{2}^{n}$ and $\mathcal{D} v=v^{n+1}-v^{n}$. Then taking the test functions $\boldsymbol{\theta}_{3}=\frac{\gamma}{\Delta t} \boldsymbol{w}_{1}^{n+1}$ and $\boldsymbol{\theta}_{4}=\frac{\gamma}{\Delta t} \boldsymbol{w}_{2}^{n+1}$, we obtain

$$
\left\{\begin{array}{l}
\frac{\gamma}{\Delta t} \int_{K} \mathcal{D} \boldsymbol{w}_{1} \cdot \boldsymbol{w}_{1}^{n+1} d K=-\frac{\gamma}{\Delta t} \int_{K} \mathcal{D} u \nabla \cdot \boldsymbol{w}_{1}^{n+1} d K+\frac{\gamma}{\Delta t} \int_{\partial K} \mathcal{D} \widehat{u} \boldsymbol{w}_{1}^{n+1} \cdot \boldsymbol{\nu} d s, \\
\frac{\gamma}{\Delta t} \int_{K} \mathcal{D} \boldsymbol{w}_{2} \cdot \boldsymbol{w}_{2}^{n+1} d K=-\frac{\gamma}{\Delta t} \int_{K} \mathcal{D} v \nabla \cdot \boldsymbol{w}_{2}^{n+1} d K+\frac{\gamma}{\Delta t} \int_{\partial K} \mathcal{D} \widehat{v} \boldsymbol{w}_{2}^{n+1} \cdot \boldsymbol{\nu} d s .
\end{array}\right.
$$

For other equations in scheme (3.24), we take the test functions as

$$
\left\{\begin{array}{l}
\varphi_{1}=r_{1}^{n}-q_{1}^{n+1}, \quad \varphi_{2}=r_{2}^{n}-q_{2}^{n+1}, \quad \boldsymbol{\theta}_{1}=-\boldsymbol{p}^{n+1}, \quad \boldsymbol{\theta}_{2}=\boldsymbol{s}^{n+1} \\
\varphi_{3}=\frac{1}{\Delta t} \mathcal{D} u, \quad \varphi_{4}=\frac{1}{\Delta t} \mathcal{D} v, \quad \varphi_{5}=-\frac{1}{\Delta t} \mathcal{D} u, \quad \varphi_{6}=-\frac{1}{\Delta t} \mathcal{D} v
\end{array}\right.
$$


Then we have,

$$
\left\{\begin{array}{l}
\frac{1}{\Delta t} \int_{K} \mathcal{D} u\left(r_{1}^{n}-q_{1}^{n+1}\right) d K=-\int_{K} \boldsymbol{s}^{n+1} \cdot \nabla\left(r_{1}^{n}-q_{1}^{n+1}\right) d K+\int_{\partial K} \widehat{\boldsymbol{s}}^{n+1} \cdot \boldsymbol{\nu}\left(r_{1}^{n}-q_{1}^{n+1}\right) d s, \\
\frac{1}{\Delta t} \int_{K} \mathcal{D} v\left(r_{2}^{n}-q_{2}^{n+1}\right) d K=-\int_{K} \frac{b\left(u^{n}, v^{n}\right)}{\rho}\left(r_{2}^{n}-q_{2}^{n+1}\right)^{2} d K, \\
-\int_{K} \boldsymbol{s}^{n+1} \cdot \boldsymbol{p}^{n+1} d K=-\int_{K} b\left(u^{n}, v^{n}\right) \boldsymbol{p}^{n+1} \cdot \boldsymbol{p}^{n+1} d K, \\
\int_{K} \boldsymbol{p}^{n+1} \cdot \boldsymbol{s}^{n+1} d K=-\int_{K}\left(r_{1}^{n}-q_{1}^{n+1}\right) \nabla \cdot \boldsymbol{s}^{n+1} d K+\int_{\partial K}\left(\widehat{r}_{1}^{n}-\widehat{q}_{1}^{n+1}\right) \boldsymbol{s}^{n+1} \cdot \boldsymbol{\nu} d s, \\
\frac{1}{\Delta t} \int_{K} q_{1}^{n+1} \mathcal{D} u d K=-\frac{\gamma}{\Delta t} \int_{K} \boldsymbol{w}_{1}^{n+1} \cdot \nabla \mathcal{D} u d K+\frac{\gamma}{\Delta t} \int_{\partial K} \widehat{\boldsymbol{w}}_{1}^{n+1} \cdot \boldsymbol{\nu} \mathcal{D} u d s, \\
\frac{1}{\Delta t} \int_{K} q_{2}^{n+1} \mathcal{D} v d K=-\frac{\gamma}{\Delta t} \int_{K} \boldsymbol{w}_{2}^{n+1} \cdot \nabla \mathcal{D} v d K+\frac{\gamma}{\Delta t} \int_{\partial K} \widehat{\boldsymbol{w}}_{2}^{n+1} \cdot \boldsymbol{\nu} \mathcal{D} v d s, \\
-\frac{1}{\Delta t} \int_{K} r_{1}^{n} \mathcal{D} u d K=-\frac{1}{\Delta t} \int_{K} \Psi_{u}\left(u^{n}, v^{n}\right) \mathcal{D} u d K, \\
\frac{1}{\Delta t} \int_{K} r_{2}^{n} \mathcal{D} v d K=-\frac{1}{\Delta t} \int_{K} \Psi_{v}\left(u^{n}, v^{n}\right) \mathcal{D} v d K .
\end{array}\right.
$$

By adding equations (3.30)-(3.31), and after a careful calculation we obtain

$$
\begin{aligned}
& \frac{\gamma}{\Delta t} \int_{\Omega}\left(\mathcal{D} \boldsymbol{w}_{1}^{n+1} \cdot \boldsymbol{w}_{1}^{n+1}+\mathcal{D} \boldsymbol{w}_{2}^{n+1} \cdot \boldsymbol{w}_{2}^{n+1}\right) d \boldsymbol{x}+\int_{\Omega} \frac{b\left(u^{n}, v^{n}\right)}{\rho}\left(\left(r_{2}^{n}-q_{2}^{n+1}\right)^{2}+\boldsymbol{p}^{n+1} \cdot \boldsymbol{p}^{n+1}\right) d \boldsymbol{x} \\
& +\frac{1}{\Delta t} \int_{\Omega} \Psi_{u}\left(u^{n}, v^{n}\right) \mathcal{D} u d \boldsymbol{x}-\frac{1}{\Delta t} \int_{\Omega} \Psi_{v}\left(u^{n}, v^{n}\right) \mathcal{D} v d \boldsymbol{x}=0 .
\end{aligned}
$$

with the help of the alternating numerical fluxes (3.28) and the Neumann bounday condition (3.22). Notice that

$$
\begin{aligned}
\Psi_{u}\left(u^{n}, v^{n}\right) \mathcal{D} u-\Psi_{v}\left(u^{n}, v^{n}\right) \mathcal{D} v & =\frac{\alpha}{2}\left(1-2 u^{n}\right) \mathcal{D} u-\beta v^{n} \mathcal{D} v \\
& =\Psi\left(u^{n+1}, v^{n+1}\right)-\Psi\left(u^{n}, v^{n}\right)+\frac{\alpha}{2}\left(u^{n+1}-u^{n}\right)^{2}+\frac{\beta}{2}\left(v^{n+1}-v^{n}\right)^{2}
\end{aligned}
$$

Therefore we have

$$
\frac{\gamma}{\Delta t} \int_{\Omega}\left(\mathcal{D} \boldsymbol{w}_{1}^{n+1} \cdot \boldsymbol{w}_{1}^{n+1}+\mathcal{D} \boldsymbol{w}_{2}^{n+1} \cdot \boldsymbol{w}_{2}^{n+1}\right) d \boldsymbol{x}+\frac{1}{\Delta t} \int_{\Omega}\left(\Psi\left(u^{n+1}, v^{n+1}\right)-\Psi\left(u^{n}, v^{n}\right)\right) d \boldsymbol{x} \leq 0
$$

which implies the energy stability result. 


\subsubsection{Algorithm flowchart and the multigrid solver}

Given $u^{n}, v^{n}$ and $q_{1}^{n}$, the algorithm to get $u^{n+1}, v^{n+1}$ and $q_{1}^{n+1}$ is as follows

1. We choose a local basis in cell $K$, then $r_{1}^{n}, r_{2}^{n}, \boldsymbol{w}_{1}^{n+1}, \boldsymbol{w}_{2}^{n+1}, q_{2}^{n+1}, \boldsymbol{p}^{n+1}, \boldsymbol{s}^{n+1}$ can be eliminated from equations (3.27c), (3.27d), (3.27a), (3.27b), (3.26) and (3.25) respectively, by simply inverting a small mass matrix in each case.

2. We get a system of 3 coupled linear equations for $\left\{u^{n+1}, v^{n+1}, q_{1}^{n+1}\right\}$

$$
\left\{\begin{array}{l}
u^{n+1}=L_{1}\left(q_{1}^{n+1}, u^{n}, v^{n}\right), \\
q_{1}^{n+1}=L_{2}\left(u^{n+1}\right), \\
v^{n+1}=L_{3}\left(v^{n+1}, u^{n}, v^{n}\right) .
\end{array}\right.
$$

3. We solve the system of nonlinear equations (3.32) at each time step by multigrid solver and get $u^{n+1}, v^{n+1}$ and $q_{1}^{n+1}$.

Remark 3.7. Observe here that we keep the variable $q_{1}$ related to $u$ since the evolution equation for $u$ contains fourth order spatial derivatives, whereas the evolution equation for $v$ has second order spatial derivatives. Hence the system $\left(u, v, q_{1}\right)$ now contains only second order derivatives.

\subsubsection{The spectral deferred correction method}

For the Allen-Cahn/Cahn-Hilliard system with constant mobility, i.e. $b(u, v)$ is constant, we can apply the semi-implicit SDC method based on the convex splitting scheme (3.23) to achieve high order temporal accuracy. An advantage of the SDC method is that it is a one step method and can be constructed easily and systematically for any order of accuracy. For convenience, the convex splitting scheme (3.23) can be rewritten as

$$
\left\{\begin{array}{l}
u^{n+1}=u^{n}+\Delta t\left(F_{S}\left(u^{n+1}\right)+F_{N}\left(u^{n}, v^{n}\right)\right), \\
v^{n+1}=v^{n}+\Delta t\left(G_{S}\left(v^{n+1}\right)+G_{N}\left(u^{n}, v^{n}\right)\right),
\end{array}\right.
$$

where $F_{S}, G_{S}$ represent the implicit part and $F_{N}, G_{N}$ represent the explicit part of the convex splitting scheme, which means

$$
\left\{\begin{array}{l}
F_{S}(u)=-\gamma \Delta^{2} u, \quad F_{N}(u, v)=\Delta\left(\Psi_{u}(u, v)\right), \\
G_{S}(v)=\gamma \Delta v, \quad G_{N}(u, v)=-\Psi_{v}(u, v) .
\end{array}\right.
$$


Then the semi-implicit SDC method can be applied iteratively to achieve high order temporal accuracy. For a detailed description of the SDC method as well as their implementation and applications, we refer the readers to $[9,13,19,21]$.

\subsubsection{The high order semi-implicit Runge-Kutta method}

For the Allen-Cahn/Cahn-Hilliard system with degenerate mobility, i.e. $b(u, v)$ is not constant, we know that the stiff and non-stiff components can not be well separated. In this case, the semi-implicit SDC method is not efficient any more. Therefore, we adopt the high order semi-implicit Runge-Kutta method. And to apply our semi-implicit scheme (2.6)-(2.8), we write the Allen-Cahn/Cahn-Hilliard system (3.19) in the form of (2.1) with $u=\left(u_{1}, u_{2}\right)$ the component treated explicitly, $v=\left(v_{1}, v_{2}\right)$ the component treated implicitly and

$$
\mathcal{H}_{A C C H}(t, u, v)=\left\{\begin{array}{l}
\nabla \cdot\left[b\left(u_{1}, u_{2}\right) \nabla\left(\Psi_{u}\left(u_{1}, u_{2}\right)-\gamma \Delta v_{1}\right)\right] \\
-\frac{1}{\rho} b\left(u_{1}, u_{2}\right)\left[\Psi_{v}\left(u_{1}, u_{2}\right)-\gamma \Delta v_{2}\right]
\end{array}\right.
$$

The semi-implicit scheme is based on the convex splitting scheme (3.23), high order accurate in time and expected to be stable with a larger time step comparing with explicit methods, which is very efficient, especially for long time simulations in multi-dimensional case.

Remark 3.8. The energy stabilities of these semi-discrete LDG schemes were proved for surface diffusion and Willmore flow of graphs [22], Cahn-Hilliard equation [18] and AllenCahn/Cahn-Hilliard system [20]. As we know, based on a convex splitting principle of the energy, the unconditional energy stability of the fully-discrete LDG scheme for Cahn-Hilliard equation was presented in [11], and for Allen-Cahn/Cahn-Hilliard system, the corresponding energy stability is given in Proposition 3.1. While for the fully-discrete LDG schemes for surface diffusion and Willmore flow of graphs, these two equations are highly nonlinear, therefore it is difficult to develop energy stable fully-discrete LDG schemes based on some splitting technique, which will be left to our future work.

Example 3.9. We consider the Allen-Cahn/Cahn-Hilliard system (3.19) with $\Psi(u, v)=$ $u-u^{2}-\frac{1}{2} v^{2}, b(u, v)=\left(1+u^{2}\right)\left(1+v^{2}\right)$ in the domain $\Omega=[0,4 \pi]$ and with periodic boundary condition. We test our method taking the exact solution

$$
u(x, t)=e^{-0.5 t} \sin (x), \quad v(x, t)=e^{-t} \cos (0.5 x)
$$


for equation (3.19) with a source term $f$, which is a given function so that (3.36) is the exact solution. When the piecewise $\mathcal{P}^{1}$ elements are used in the LDG method, the second order semi-implicit Runge-Kutta method is used for time integration, while for $\mathcal{P}^{2}$ approximation, we adopt the third order scheme. The $L^{2}$ and $L^{\infty}$ errors and the numerical orders of accuracy at time $T=0.5$ are contained in Table 3.4, which shows the $(k+1)$-th order of accuracy in both $L^{2}$ and $L^{\infty}$ norms for $\mathcal{P}^{k}$ approximation. Figure 3.10 shows the near optimal complexity of the multigrid solver for the Allen-Cahn/Cahn-Hilliard system, which means that the convergence is nearly independent of the grid size $\Delta x$.

\begin{tabular}{|c|c|cc|cc|cc|cc|}
\hline & & \multicolumn{5}{|c|}{$u$} & \multicolumn{5}{c|}{$v$} \\
\hline \multirow{4}{*}{$\mathcal{P}^{1}$} & $N$ & $L^{2}$ error & order & $L^{\infty}$ error & order & $L^{2}$ error & order & $L^{\infty}$ error & order \\
& 16 & $7.61 \mathrm{E}-02$ & - & $5.82 \mathrm{E}-02$ & - & $1.91 \mathrm{E}-02$ & - & $9.79 \mathrm{E}-03$ & - \\
& 32 & $1.85 \mathrm{E}-02$ & 2.03 & $1.57 \mathrm{E}-02$ & 1.89 & $4.28 \mathrm{E}-03$ & 2.16 & $2.39 \mathrm{E}-03$ & 2.03 \\
& 64 & $4.59 \mathrm{E}-03$ & 2.01 & $3.99 \mathrm{E}-03$ & 1.98 & $1.03 \mathrm{E}-03$ & 2.04 & $6.12 \mathrm{E}-04$ & 1.96 \\
& 128 & $1.14 \mathrm{E}-03$ & 2.00 & $1.00 \mathrm{E}-03$ & 2.00 & $2.57 \mathrm{E}-04$ & 2.01 & $1.53 \mathrm{E}-04$ & 1.99 \\
\hline \multirow{4}{*}{$\mathcal{P}^{2}$} & 16 & $4.70 \mathrm{E}-03$ & - & $4.13 \mathrm{E}-03$ & - & $5.77 \mathrm{E}-04$ & - & $3.84 \mathrm{E}-04$ & - \\
& 32 & $5.80 \mathrm{E}-04$ & 3.01 & $4.91 \mathrm{E}-04$ & 3.07 & $7.54 \mathrm{E}-05$ & 2.93 & $4.84 \mathrm{E}-05$ & 2.99 \\
& 64 & $7.27 \mathrm{E}-05$ & 2.99 & $6.14 \mathrm{E}-05$ & 2.99 & $1.02 \mathrm{E}-05$ & 2.87 & $6.27 \mathrm{E}-06$ & 2.94 \\
& 128 & $9.38 \mathrm{E}-06$ & 2.96 & $7.94 \mathrm{E}-06$ & 2.95 & $1.53 \mathrm{E}-06$ & 2.74 & $8.56 \mathrm{E}-07$ & 2.87 \\
\hline
\end{tabular}

Table 3.4: Accuracy test for the Allen-Cahn/Cahn-Hilliard system (3.19) with the exact solution (3.36) at time $T=0.5$.

Example 3.10. To demonstrate the theoretical result of unconditional energy stability for the Allen-Cahn/Cahn-Hilliard system, we present an example here. We consider the system (3.19) in $\Omega=[0,2 \pi] \times[0,2 \pi]$ with Neumann boundary conditions $(3.22), \gamma=1, \theta=0$, $\alpha=1, \beta=1, \rho=1$ with degenerate and constant mobility respectively. For the tests we take the exact solution of

$$
u(x, y, t)=e^{-2 t} \cos (x) \cos (y), \quad v(x, y, t)=\frac{1}{2} \cos (x) \cos (y),
$$

with the source terms $f(x, y, t)$ and $g(x, y, t)$, respectively. We consider the following cases:

1. With degenerate mobility $b(u, v)=\left(1-u^{2}\right)\left(0.25-v^{2}\right)$,

2. With constant mobility $b=0.25$, which is the maximum of the degenerate mobility. 


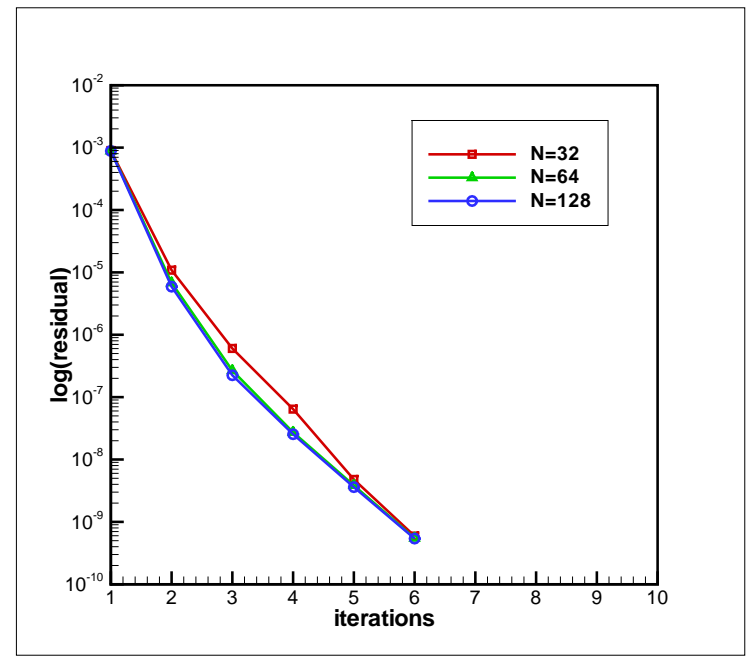

(a) $\mathcal{P}^{1}$ approximation

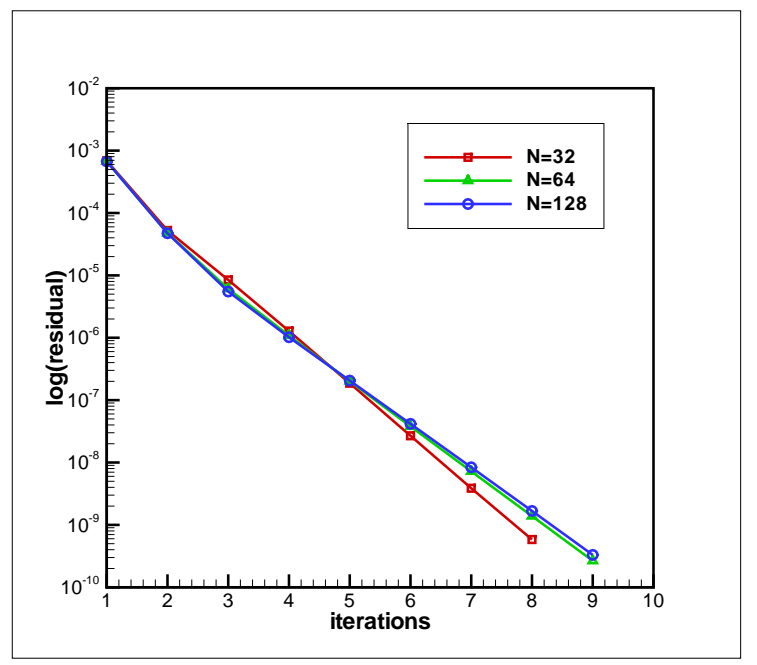

(b) $\mathcal{P}^{2}$ approximation

Figure 3.10: Convergence rates of multigrid solver with $\mathcal{P}^{1}$ and $\mathcal{P}^{2}$ approximation for AllenCahn/Cahn-Hilliard system.

To obtain high order accuracy scheme in both space and time, we choose $\mathcal{P}^{k}$ approximation for spatial discretization, semi-implicit SDC method and semi-implicit Runge-Kutta method for constant mobility and degenerate mobility, respectively. Table 3.5 and Table 3.6 show the $L^{2}$ and $L^{\infty}$ errors and numerical orders of accuracy at time $T=0.5$. We can see that these two cases with $\mathcal{P}^{k}$ approximation give $(k+1)$-th order of accuracy.

We present in Figure 3.11 and Figure 3.12 the time evolution of the energy $\mathcal{E}_{A C C H}(t)$ (which is defined in (3.21)) of the numerical solution and its dissipation, and the dissipation $\mathcal{I}_{A C C H}(t)$ is defined by

$$
\mathcal{I}_{A C C H}(t)=\int_{\Omega} b(u, v)\left(\left|\nabla \mu_{1}\right|^{2}+\frac{1}{\rho}\left(\mu_{2}\right)^{2}\right) d \boldsymbol{x},
$$

with $\mu_{1}=-\gamma \Delta u+\Psi_{u}$ and $\mu_{2}=-\gamma \Delta v+\Psi_{v}$, which show that our schemes are stable numerically.

Finally, we remark that the LDG spatial discretization does allow for more flexibility than that of the finite difference method in several other ways. LDG methods are a class of finite element methods, which can handle the irregular computational domain and complex boundary conditions easily comparing with the finite difference methods. Meanwhile, since the basis functions can be completely discontinuous, discontinuous Galerkin methods have the flexibility which is not shared by typical finite element methods, such as the allowance of arbitrary triangulation with hanging nodes, complete freedom in changing the polynomial 


\begin{tabular}{|c|c|cc|cc|cc|cc|}
\hline & & \multicolumn{6}{|c|}{$u$} & \multicolumn{5}{c|}{$v$} \\
\hline & $N$ & $L^{2}$ error & order & $L^{\infty}$ error & order & $L^{2}$ error & order & $L^{\infty}$ error & order \\
\hline \multirow{4}{*}{$\mathcal{P}^{1}$} & 16 & $2.20 \mathrm{E}-02$ & - & $1.52 \mathrm{E}-02$ & - & $1.10 \mathrm{E}-02$ & - & $8.42 \mathrm{E}-03$ & - \\
& 32 & $5.45 \mathrm{E}-03$ & 2.01 & $3.81 \mathrm{E}-03$ & 2.00 & $2.72 \mathrm{E}-03$ & 2.01 & $2.05 \mathrm{E}-03$ & 2.03 \\
& 64 & $1.35 \mathrm{E}-03$ & 2.01 & $9.46 \mathrm{E}-04$ & 2.01 & $6.76 \mathrm{E}-04$ & 2.01 & $4.88 \mathrm{E}-04$ & 2.07 \\
\hline \multirow{4}{*}{$\mathcal{P}^{2}$} & 16 & $1.33 \mathrm{E}-03$ & - & $1.31 \mathrm{E}-03$ & - & $6.68 \mathrm{E}-04$ & - & $6.72 \mathrm{E}-04$ & - \\
& 32 & $1.68 \mathrm{E}-04$ & 2.99 & $1.60 \mathrm{E}-04$ & 3.03 & $8.40 \mathrm{E}-05$ & 2.99 & $8.03 \mathrm{E}-05$ & 3.06 \\
& 64 & $2.10 \mathrm{E}-05$ & 2.99 & $1.98 \mathrm{E}-05$ & 3.01 & $1.05 \mathrm{E}-05$ & 2.99 & $9.91 \mathrm{E}-06$ & 3.01 \\
\hline
\end{tabular}

Table 3.5: Accuracy test for the Allen-Cahn/Cahn-Hilliard system (3.19) with degenerate mobility at time $T=0.5$ in $2 D$.

\begin{tabular}{|c|c|cc|cc|cc|cc|}
\hline & & \multicolumn{6}{|c|}{$u$} & \multicolumn{5}{c|}{$v$} \\
\hline & $N$ & $L^{2}$ error & order & $L^{\infty}$ error & order & $L^{2}$ error & order & $L^{\infty}$ error & order \\
\hline \multirow{4}{*}{$\mathcal{P}^{1}$} & 16 & $2.23 \mathrm{E}-02$ & - & $1.51 \mathrm{E}-02$ & - & $1.11 \mathrm{E}-02$ & - & $8.22 \mathrm{E}-03$ & - \\
& 32 & $5.47 \mathrm{E}-03$ & 2.02 & $3.80 \mathrm{E}-03$ & 1.99 & $2.73 \mathrm{E}-03$ & 2.02 & $1.95 \mathrm{E}-03$ & 2.07 \\
& 64 & $1.35 \mathrm{E}-03$ & 2.01 & $9.49 \mathrm{E}-04$ & 2.00 & $6.76 \mathrm{E}-04$ & 2.01 & $4.77 \mathrm{E}-04$ & 2.03 \\
\hline \multirow{4}{*}{$\mathcal{P}^{2}$} & 16 & $1.33 \mathrm{E}-03$ & - & $1.30 \mathrm{E}-03$ & - & $6.68 \mathrm{E}-04$ & - & $6.70 \mathrm{E}-04$ & - \\
& 32 & $1.68 \mathrm{E}-04$ & 2.99 & $1.60 \mathrm{E}-04$ & 3.02 & $8.40 \mathrm{E}-05$ & 2.99 & $8.03 \mathrm{E}-05$ & 3.06 \\
& 64 & $2.10 \mathrm{E}-05$ & 2.99 & $1.98 \mathrm{E}-05$ & 3.01 & $1.05 \mathrm{E}-05$ & 2.99 & $9.91 \mathrm{E}-06$ & 3.01 \\
\hline
\end{tabular}

Table 3.6: Accuracy test for the Allen-Cahn/Cahn-Hilliard system (3.19) with constant mobility at time $T=0.5$ in $2 D$.

degrees in each element independent of that in the neighbors ( $p$-adaptivity), and extremely local data structure (elements only communicate with immediate neighbors regardless of the order of accuracy of the scheme) and the resulting embarrassingly high parallel efficiency.

\section{Conclusion}

In this paper, we have explored a high order semi-implicit Runge-Kutta method for solving the ODEs resulting from a local discontinuous Galerkin spatial discretization to highly nonlinear PDEs containing higher order spatial derivatives, which consist of the surface diffusion and Willmore flow of graphs, the Cahn-Hilliard equation and the Allen-Cahn/Cahn-Hilliard system. With the proposed semi-implicit temporal method, the severe time step restriction of explicit methods can be relaxed and we can achieve high order temporal accuracy with a 


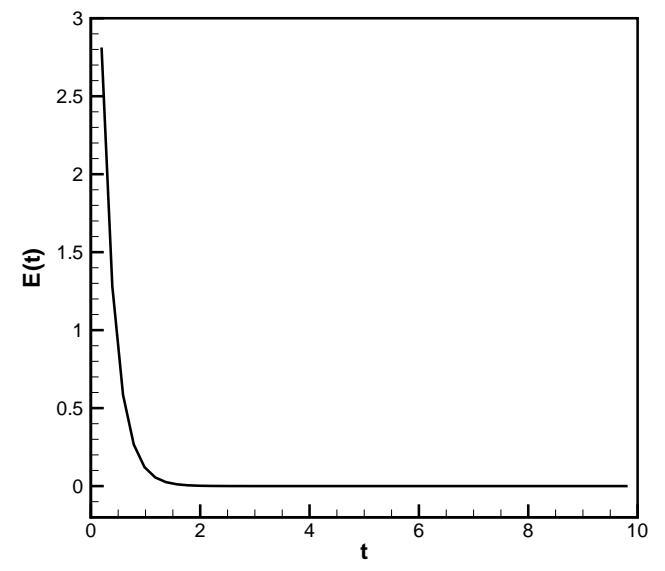

(a) functional $\mathcal{E}_{A C C H}(t)$

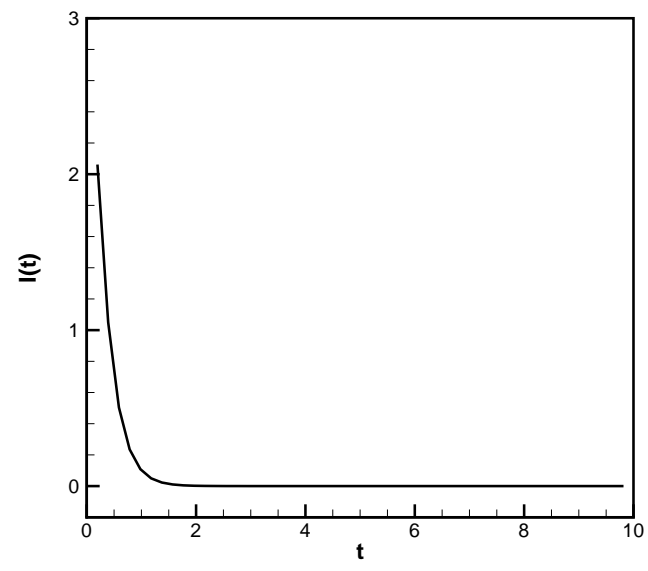

(b) dissipation $\mathcal{I}_{A C C H}(t)$

Figure 3.11: Evolution of the energy and the dissipation for Allen-Cahn/Cahn-Hilliard system with degenerate mobility in $2 D$.

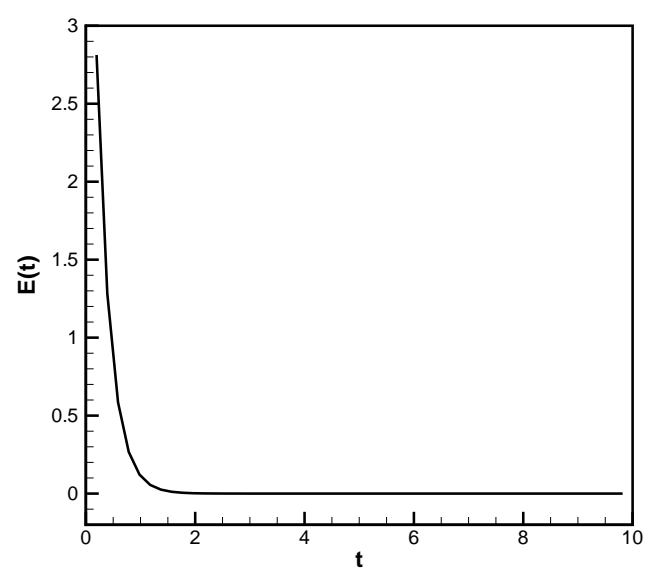

(a) functional $\mathcal{E}_{A C C H}(t)$

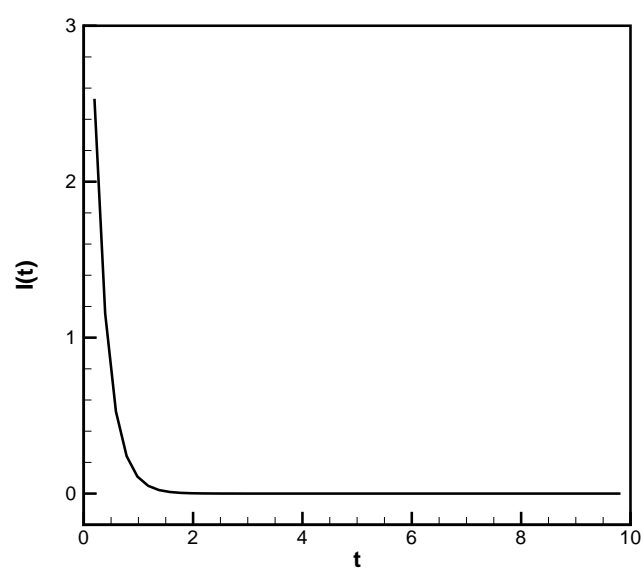

(b) dissipation $\mathcal{E}_{A C C H}(t)$

Figure 3.12: Evolution of the energy and the dissipation for Allen-Cahn/Cahn-Hilliard system with constant mobility in $2 D$.

larger time step. However, the equations at the implicit time level are linear or nonlinear, and to enhance the efficiency of the solver, we employed the linear and nonlinear multigrid solver to solve algebraic equations, respectively. In addition, we have developed a first order fully discrete LDG scheme for the Allen-Cahn/Cahn-Hilliard system and proved the unconditional energy stability. Numerically we show the high order accuracy of the proposed 
schemes, in both time and space, with a larger time step. Also, the long time simulations show the capacity and efficiency of the proposed temporal and spatial methods.

\section{References}

[1] F. Bassi and S. Rebay, A high-order accurate discontinuous finite element method for the numerical solution of the compressible Navier-Stokes equations, J. Comput. Phys., 131, (1997), pp.267-279.

[2] S. Boscarino, F. Filbet and G. Russo, High order semi-implicit schemes for time dependent partial differential equations, submitted.

[3] B. Cockburn and C.-W. Shu, TVB Runge-Kutta local projection discontinuous Galerkin finite element method for conservation laws II: general framework, Math. Comp., 52, (1989), pp.411-435.

[4] B. Cockburn, S.-Y. Lin and C.-W. Shu, TVB Runge-Kutta local projection discontinuous Galerkin finite element method for conservation laws III: one dimensional systems, J. Comput. Phys., 84, (1989), pp.90-113.

[5] B. Cockburn, S. Hou and C.-W. Shu, The Runge-Kutta local projection discontinuous Galerkin finite element method for conservation laws $I V$ : the multidimensional case, Math. Comp., 54, (1990), pp.545-581.

[6] B. Cockburn and C.-W. Shu, The Runge-Kutta discontinuous Galerkin method for conservation laws V: multidimensional systems, J. Comput. Phys., 141, (1998), pp.199-224.

[7] B. Cockburn and C.-W. Shu, The local discontinuous Galerkin method for timedependent convection-diffusion systems, SIAM J. Numer. Anal., 35, (1998), pp.24402463.

[8] K. Deckelnick and G. Dziuk, Error analysis of a finite element method for the Willmore flow of graphs, Interfaces Free Bound., 8, (2006), pp.21-46.

[9] A. Dutt, L. Greengard and V. Rokhlin, Spectral deferred correction methods for ordinary differential equations, BIT, 40, (2000), pp.241-266. 
[10] D.J. Eyre, Unconditionally gradient stable time marching the Cahn-Hilliard equation, in: J.W. Bullard, R. Kalia, M. Stoneham, L.Q. Chen (Eds), Computational and Mathematical Models of Microstructural Evolution, vol. 53, Materials Research Society, Warrendale, 1998, pp.1685-1712.

[11] R. Guo and Y. Xu, Efficient solvers of discontinuous Galerkin discretization for the Cahn-Hilliard equations, J. Sci. Comput., 58, (2014), pp.380-408.

[12] E. Hairer and G. Wanner, Solving Ordinary Differential Equation. II. Stiff and differential algebraic problems, Springer Series in Comput. Mathematics, Vol. 14, Springer Verlag, (2nd edition), 1996.

[13] M.L. Minion,Semi-implicit spectral deferred correction methods for ordinary differential equations, Math. Sci., 1, (2003), pp.471-500.

[14] L. Pareschi and G. Russo,Implicit-Explicit Runge-Kutta schemes for stiff systems of differential equations, Recent trends in numerical analysis, pp.269-288, Adv. Theory Comput. Math. 3, Nova Sci. Publ. Huntington, NY, (2001).

[15] W.H. Reed and T.R. Hill, Triangular mesh method for the neutron transport equation, Technical report LA-UR-73-479, Los Alamos Scientific Laboratory, Los Alamos, NM, 1973.

[16] P. Smereka, Semi-implicit level set methods for curvature and surface diffusion motion, J. Sci. Comput., 19, (2003), pp.439-456.

[17] U. Trottenberg, C. Oosterlee and A. Schüller, Multigrid, Academic Press, New York (2005).

[18] Y. Xia, Y. Xu and C.-W. Shu, Local discontinuous Galerkin methods for the CahnHilliard type equations, J. Comput. Phys., 227, (2007), pp.677-693.

[19] Y. Xia, Y. Xu and C.-W. Shu, Efficient time discretization for local discontinuous Galerkin methods, Discrete Contin. Dyn. Syst. Ser. B, 8, (2007), pp.677-693.

[20] Y. Xia, Y. Xu and C.-W. Shu, Application of the local discontinuous Galerkin method for the Allen-Cahn/Cahn-Hilliard system, Commun. Comput. Phys., 5, (2009), pp.821-835. 
[21] Y. Xia, A fully discrete stable discontinuous Galerkin method for the thin film epitaxy problem without slope selection, J. Comput. Phys., 280, (2015), pp.248-260.

[22] Y. Xu and C.-W. Shu, Local discontinuous Galerkin method for surface diffusion and Willmore flow of graphs, J. Sci. Comput., 40, (2009), pp.375-390.

[23] Y. Xu and C.-W. Shu, Local discontinuous Galerkin methods for high-order timedependent partial differential equations, Commun. Comput. Phys., 7, (2010), pp.1-46. 